\title{
Probing unified theories with reduced couplings at future hadron colliders
}

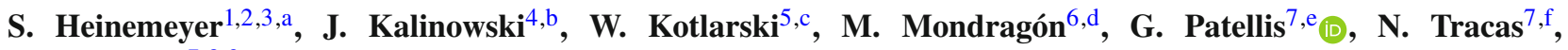 \\ G. Zoupanos $7,8,9, \mathrm{~g}$ \\ ${ }^{1}$ Instituto de Física Teórica (UAM/CSIC), Universidad Autónoma de Madrid Cantoblanco, 28049 Madrid, Spain \\ ${ }^{2}$ Campus of International Excellence UAM + CSIC, Cantoblanco, 28049 Madrid, Spain \\ ${ }^{3}$ Instituto de Física de Cantabria (CSIC-UC), 39005 Santander, Spain \\ ${ }^{4}$ Faculty of Physics, University of Warsaw, ul. Pasteura 5, 02-093 Warsaw, Poland \\ ${ }^{5}$ Technische Universität Dresden, Institut für Kern- und Teilchenphysik (IKTP), 01069 Dresden, Germany \\ ${ }^{6}$ Instituto de Física, Universidad Nacional Autónoma de México, A.P. 20-364, 01000 Mexico, CDMX, Mexico \\ ${ }^{7}$ Physics Department, Nat. Technical University, 15780 Zografou, Athens, Greece \\ ${ }^{8}$ Max-Planck Institut für Physik, Föhringer Ring 6, 80805 München, Germany \\ ${ }^{9}$ Theoretical Physics Department, CERN, Geneva, Switzerland
}

Received: 23 November 2020 / Accepted: 7 February 2021 / Published online: 23 February 2021

(C) The Author(s) 2021

\begin{abstract}
The search for renormalization group invariant relations among parameters to all orders in perturbation theory constitutes the basis of the reduction of couplings concept. Reduction of couplings can be achieved in certain $N=1$ supersymmetric grand unified theories and few of them can become even finite at all loops. We review the basic idea, the tools that have been developed as well as the resulting theories in which successful reduction of couplings has been achieved so far. These include: (i) a reduced version of the minimal $N=1 S U(5)$ model, (ii) an all-loop finite $N=1 S U(5)$ model, (iii) a two-loop finite $N=1 S U(3)^{3}$ model and finally (vi) a reduced version of the Minimal Supersymmetric Standard Model. In this paper we present a number of benchmark scenarios for each model and investigate their observability at existing and future hadron colliders. The heavy supersymmetric spectra featured by each of the above models are found to be beyond the reach of the 14 TeV HL-LHC. It is also found that the reduced version of the MSSM is already ruled out by the LHC searches for heavy neutral MSSM Higgs bosons. In turn the discovery potential of the $100 \mathrm{TeV}$ FCC-hh is investigated and found that large parts of the predicted spectrum of these models can be tested,
\end{abstract}

\footnotetext{
a e-mail: Sven.Heinemeyer@cern.ch

b e-mail: kalino@fuw.edu.pl

c e-mail: wojciech.kotlarski@tu-dresden.de

de-mail: myriam@fisica.unam.mx

ee-mail: patellis@central.ntua.gr (corresponding author)

fe-mail: ntrac@central.ntua.gr

g e-mail: George.Zoupanos@ cern.ch
}

but the higher mass regions are beyond the reach even of the FCC-hh.

\section{Introduction}

The reduction of couplings method [1-4] (see also [5-8]) is a promising method which relates originally seemingly independent parameters to a single, "primary" coupling. The method requires the original theory to which it is applied to be a renormalizable one, and the resulting relation among the parameters to be valid at all energy scales, i.e. renormalization group invariant (RGI).

A next (natural) step, after the introduction of a novel symmetry through a grand unified theory (GUT) [9-14]), in order to achieve reduction of free parameters of the SM is the relation of the gauge sector to the Yukawa sector (gauge Yukawa unification, GYU). This was the central characteristic of the reduction of couplings approach in the first period of searches, applied in $N=1$ GUTs [15-28]. According to that approach, being in a GUT environment, RGI relations are set between the unification scale and the Planck scale. One-loop consideration can guarantee the all-loop validity of those relations. Moreover, RGI relations can be found which guarantee all order finiteness of a theory. The method has predicted the top quark mass in the finite $N=1 S U(5)$ model $[15,16]$ as well as in the minimal $N=1 S U(5)$ one [17] before its experimental measurement [29].

Since SuperSymmetry (SUSY) seems an essential ingredient for the reduction of couplings method, we have to 
include a supersymmetry breaking sector (SSB), which involves dimension- 1 and -2 couplings. The supergraph method and the spurion superfield technique played an important role for the progress in that sector, leading to complete all-loop finite models, i.e. including the SSB sector. The all-loop finite $N=1 S U(5)$ model [30] has given a prediction for the Higgs mass compatible with the experimental results [31-33] and a heavy SUSY mass spectrum, consistent with the experimental non-observation of these particles. The reduction of couplings method has been applied to several other cases. The full analysis of the most successful models, that includes predictions in agreement with the experimental measurements of the top and bottom quark masses for each model, can be found in a recent work [44].

In this paper we address the question to what extent the reduction of couplings idea, as applied in the so far phenomenologically successful models, can be experimentally tested at HL-LHC and future FCC hadron collider. To this end we propose a number of benchmark points for each model. We present the SUSY breaking parameters used as input in each benchmark to calculate the corresponding Higgs boson and supersymmetric particles masses. Then we compute the expected production cross sections at the $14 \mathrm{TeV}$ (HL-)LHC and the $100 \mathrm{TeV}$ FCC-hh and investigate which production channels can be observed.

The present work is organized as follows. In Sect. 2 we review the basic idea of the reduction of couplings. In Sect. 3 we list the phenomenological constraints used in our analyses, wile in Sect. 4 we explain the computational setup. In Sects. 5-8 wereview four interesting models, namely (i) the minimal $N=1$ supersymmetric $S U$ (5), (ii) the finite $N=1$ supersymmetric $S U(5)$, (iii) the finite $S U(3)^{3}$ and (iv) the MSSM, in which the reduction of couplings has been successfully applied. We briefly review some earlier results of our phenomenological analysis. In this context the new version of the FeynHiggs [34-43] code plays a crucial role, which was used to calculate the Higgs-boson predictions, in particular the mass of the lightest CP-even Higgs boson. The improved predictions of FeynHiggs are compared with the LHC measurements and the Beyond Standard Model (BSM) Higgs boson searches. As a new part of the analysis we examine in each model the discovery potential of the Higgs and SUSY spectrum at approved future and hypothetical future hadron colliders. Finally, Sect. 8 is dedicated to brief conclusive remarks.

\section{Theoretical basis}

Here we will briefly review the core idea of the reduction of couplings method. The target is to single out a basic parameter (which we will call the primary coupling), where all other parameters can be expressed in terms of this one through
RGI relations. Such a relation has, in general, the form $\Phi\left(g_{1}, \ldots, g_{A}\right)=$ const. which should satisfy the following partial differential equation (PDE)

$\mu \frac{d \Phi}{d \mu}=\vec{\nabla} \Phi \cdot \vec{\beta}=\sum_{a=1}^{A} \beta_{a} \frac{\partial \Phi}{\partial g_{a}}=0$

where $\beta_{a}$ is the $\beta$-functions of $g_{a}$. The above PDE is equivalent to the following set of ordinary differential equations (ODEs), which are called reduction equations (REs) [2-4],

$\beta_{g} \frac{d g_{a}}{d g}=\beta_{a}, \quad a=1, \ldots, A-1$,

where now $g$ and $\beta_{g}$ are the primary coupling and its corresponding $\beta$-function. There are obviously $A-1$ relations in the form of $\Phi\left(g_{1}, \ldots, g_{A}\right)=$ const. in order to express all other couplings in term of the primary one.

The crucial demand is that the above REs admit power series solutions

$g_{a}=\sum_{n} \rho_{a}^{(n)} g^{2 n+1}$,

which preserve perturbative renormalizability. Without this requirement, we just trade each "dependent" coupling for an integration constant. The power series, which are a set of special solutions, fix that constant. It is very important to point out that the uniqueness of such a solution can be already decided at the one-loop level [2-4]. In supersymmetric theories, where the asymptotic behaviour of several parameters are similar, the use of power series as solutions of the REs are justified. But, usually, the reduction is not "complete", which means that not all of the couplings can be reduced in favor of the primary one, leading to the so called "partial reduction" $[45,46]$.

We proceed to the reduction scheme for massive parameters, which is far from being straightforward. A number of conditions is required (see for example [47]). Nevertheless, progress has been achieved, starting from [48], and finally we can introduce mass parameters and couplings carrying mass dimension $[49,50]$ in the same way as dimensionless couplings.

Consider the superpotential

$W=\frac{1}{2} \mu^{i j} \Phi_{i} \Phi_{j}+\frac{1}{6} C^{i j k} \Phi_{i} \Phi_{j} \Phi_{k}$,

and the SSB sector Lagrangian

$$
\begin{aligned}
-\mathcal{L}_{\mathrm{SSB}}= & \frac{1}{6} h^{i j k} \phi_{i} \phi_{j} \phi_{k}+\frac{1}{2} b^{i j} \phi_{i} \phi_{j} \\
& +\frac{1}{2}\left(m^{2}\right)_{i}^{j} \phi^{* i} \phi_{j}+\frac{1}{2} M \lambda_{i} \lambda_{i}+\text { h.c. },
\end{aligned}
$$


where $\phi_{i}$ 's are the scalar fields of the corresponding superfields $\Phi_{i}$ 's and $\lambda_{i}$ are the gauginos.

Let us write down some well known relations:

(i) The $\beta$-function of the gauge coupling at one-loop level is given by [51-55]

$\beta_{g}^{(1)}=\frac{d g}{d t}=\frac{g^{3}}{16 \pi^{2}}\left[\sum_{i} T\left(R_{i}\right)-3 C_{2}(G)\right]$,

where $T\left(R_{i}\right)$ is the Dynkin index of the rep $R_{i}$ where the matter fields belong and $C_{2}(G)$ is the quadratic Casimir operator of the adjoint rep $G$.

(ii) The anomalous dimension $\gamma_{j}^{(1)}{ }_{j}$, at a one-loop level, of a chiral superfield is

$\gamma^{(1)}{ }_{j}^{i}=\frac{1}{32 \pi^{2}}\left[C^{i k l} C_{j k l}-2 g^{2} C_{2}\left(R_{i}\right) \delta_{j}^{i}\right]$.

(iii) The $\beta$-functions of $C_{i j k}$ 's, at one-loop level, following the $N=1$ non-renormalization theorem [56-58], are expressed in terms of the anomalous dimensions of the fields involved

$\beta_{C}^{i j k}=\frac{d C_{i j k}}{d t}=C_{i j l} \gamma_{k}^{l}+C_{i k l} \gamma_{j}^{l}+C_{j k l} \gamma_{i}^{l}$.

We proceed by assuming that the REs admit power series solutions:

$C^{i j k}=g \sum_{n=0} \rho_{(n)}^{i j k} g^{2 n}$

Trying to obtain all-loop results we turn to relations among $\beta$-functions. The spurion technique [58-62] gives all-loop relations among SSB $\beta$-functions [63-69]. Then, assuming that the reduction of $C^{i j k}$ is possible to all orders

$$
\frac{d C^{i j k}}{d g}=\frac{\beta_{C}^{i j k}}{\beta_{g}}
$$

as well as for $h^{i j k}$

$$
h^{i j k}=-M \frac{d C^{i j k}}{d \ln g},
$$

it can be proven $[70,71]$ that the following relations are all-loop RGI

$$
\begin{aligned}
M & =M_{0} \frac{\beta_{g}}{g}, \\
h^{i j k} & =-M_{0} \beta_{C}^{i j k}, \\
b^{i j} & =-M_{0} \beta_{\mu}^{i j}, \\
\left(m^{2}\right)_{j}^{i} & =\frac{1}{2}\left|M_{0}\right|^{2} \mu \frac{d \gamma_{j}^{i}}{d \mu},
\end{aligned}
$$

where $M_{0}$ is an arbitrary reference mass scale to be specified and Eq. (12) is the Hisano-Shifman relation [66] (note that in both assumptions we do not rely on specific solutions of these equations).

As a next step we substitute the last equation, Eq. (15), by a more general RGI sum rule that holds to all orders [72]

$$
\begin{aligned}
m_{i}^{2} & +m_{j}^{2}+m_{k}^{2} \\
= & |M|^{2}\left\{\frac{1}{1-g^{2} C_{2}(G) /\left(8 \pi^{2}\right)} \frac{d \ln C^{i j k}}{d \ln g}+\frac{1}{2} \frac{d^{2} \ln C^{i j k}}{d(\ln g)^{2}}\right\} \\
& +\sum_{l} \frac{m_{l}^{2} T\left(R_{l}\right)}{C_{2}(G)-8 \pi^{2} / g^{2}} \frac{d \ln C^{i j k}}{d \ln g}
\end{aligned}
$$

which leads to the following one-loop relation

$m_{i}^{2}+m_{j}^{2}+m_{k}^{2}=|M|^{2}$.

Finally, note that in the case of product gauge groups, Eq. (12) takes the form

$M_{i}=\frac{\beta_{g_{i}}}{g_{i}} M_{0}$,

where $i$ denotes the group of the product. This will be used in the Reduced MSSM case.

Consider an $N=1$ globally supersymmetric gauge theory, which is chiral and anomaly free, where $G$ is the gauge group and $g$ the associated gauge coupling. The theory has the superpotential of Eq. (4), while the one-loop gauge and $C_{i j k} \mathrm{~s} \beta$-functions are given by Eqs. (6) and (8) respectively and the one-loop anomalous dimensions of the chiral superfields by Eq. (7).

Demanding the vanishing of all one-loop $\beta$-functions, Eqs. $(6,7)$ lead to the relations

$\sum_{i} T\left(R_{i}\right)=3 C_{2}(G)$,

$$
C^{i k l} C_{j k l}=2 \delta_{j}^{i} g^{2} C_{2}\left(R_{i}\right) .
$$

The finiteness conditions for an $N=1$ supersymmetric theory with $S U(N)$ associated group is found in [73] while dis- 
cussion of the no-charge renormalization and anomaly free requirements can be found in [74]. It should be noted that conditions (19) and (20) are necessary and sufficient to ensure finiteness at the two-loop level [51-55].

The requirement of finiteness, at the one-loop level, in softly broken SUSY theories demands additional constraints among the soft terms of the SSB sector [75], while, once more, these one-loop requirements assure two-loop finiteness, too [76]. These conditions impose restrictions on the irreducible representations $R_{i}$ of the gauge group $G$ as well as on the Yukawa couplings. For example, since $U(1) \mathrm{s}$ are not compatible with condition (19), the MSSM is excluded. Therefore, a GUT is initially required with the MSSM being its low energy theory. Also, since condition (20) forbids the appearance of gauge singlets $\left(C_{2}(1)=0\right)$, F-type spontaneous symmetry breaking [77] are not compatible with finiteness. Finally, D-type spontaneous breaking [78] is also incompatible since it requires a $U(1)$ group.

The nontrivial point is that the relations among couplings (gauge and Yukawa) which are imposed by the conditions (19) and (20) should hold at any energy scale. The necessary and sufficient condition is to require that such relations are solutions to the REs (see Eq. (10))

$\beta_{g} \frac{d C_{i j k}}{d g}=\beta_{i j k}$

holding at all orders. We note, once more, that the existence of one-loop level power series solution guarantees the all-order series.

There exist the following theorem $[79,80]$ which points down which are the necessary and sufficient conditions in order for an $N=1$ SUSY theory to be all-loop finite. In Refs. [7985 ] it was shown that for an $N=1$ SUSY Yang-Mills theory, based on a simple gauge group, if the following four conditions are fulfilled:

(i) No gauge anomaly is present.

(ii) The $\beta$-function of the gauge coupling is zero at one-loop level

$$
\beta_{g}^{(1)}=0=\sum_{i} T\left(R_{i}\right)-3 C_{2}(G) .
$$

(iii) The condition of vanishing for the one-loop anomalous dimensions of matter fields,

$$
\gamma_{j}^{(1) i}=0=\frac{1}{32 \pi^{2}}\left[C^{i k l} C_{j k l}-2 g^{2} C_{2}(R) \delta_{j}^{i}\right]
$$

admits solution of the form

$$
C_{i j k}=\rho_{i j k} g, \quad \rho_{i j k} \in \mathbb{C} .
$$

(iv) When considered as solutions of vanishing Yukawa $\beta$ functions (at one-loop order), i.e. $\beta_{i j k}=0$, the above solutions are isolated and non-degenerate;

then, each of the solutions in Eq. (24) can be extended uniquely to a formal power series in $g$, and the associated super Yang-Mills models depend on the single coupling constant $g$ with a vanishing, at all orders, $\beta$ function.

While the validity of the above cannot be extended to non-SUSY theories, it should be noted that reduction of couplings and finiteness are intimately related.

\section{Phenomenological constraints}

In this section we briefly review several experimental constraints that were applied in our phenomenological analysis. The used values do not correspond to the latest experimental results, which, however, has a negligible impact on our analysis.

In our models we evaluate the pole mass of the top quark while the bottom quark mass is evaluated at the $M_{Z}$ scale (to avoid uncertainties to its pole mass). The experimental values, taken from Ref. [86] are:

$m_{t}^{\text {exp }}=173.1 \pm 0.9 \mathrm{GeV}$

$m_{b}\left(M_{Z}\right)=2.83 \pm 0.10 \mathrm{GeV}$.

We interpret the Higgs-like particle discovered in July 2012 by ATLAS and CMS [31,32] as the light CP-even Higgs boson of the MSSM [87-89]. The Higgs boson experimental average mass is [86 ${ }^{1}$

$M_{h}^{\text {exp }}=125.10 \pm 0.14 \mathrm{GeV}$.

The theoretical uncertainty [34,35], however, for the prediction of $M_{h}$ in the MSSM dominates the total uncertainty, since it is much larger than the experimental one. In our following analyses we shall use the new FeynHiggs code [34-42] (Version 2.16.0) to predict the Higgs mass. ${ }^{2}$ FeynHiggs evaluates the Higgs masses based on a combination of fixed order diagrammatic calculations and resummation of the (sub)leading logarithmic contributions at all orders. This provides a reliable $M_{h}$ even for a large SUSY scale. This new version gives a downward shift on the Higgs mass $M_{h}$ of $\mathcal{O}(2 \mathrm{GeV})$ for large SUSY masses and in particular gives a reliable point-by-point evaluation of the Higgs-

\footnotetext{
1 This is the latest available LHC combination. More recent measurements confirm this value.

2 An analysis of the impact of the improved $M_{h}$ calculation in various SUSY models can be found in [90].
} 
boson mass uncertainty [43]. The theoretical uncertainty calculated is added linearly to the experimental error in Eq. (26).

Furthermore, recent results from the ATLAS experiment [91] set limits to the mass of the pseudoscalar Higgs boson, $M_{A}$, in comparison with $\tan \beta$. For models with $\tan \beta \sim$ 45-55, as the ones examined here, the lowest limit for the physical pseudoscalar Higgs mass is

$M_{A} \gtrsim 1900 \mathrm{GeV}$.

We also consider the following four flavor observables where SUSY has non-negligible impact. For the branching ratio $\mathrm{BR}(b \rightarrow s \gamma)$ we take a value from [92-97], while for the branching ratio $\operatorname{BR}\left(B_{S} \rightarrow \mu^{+} \mu^{-}\right)$we use a combination of [98-105]:

$$
\begin{aligned}
& \frac{\mathrm{BR}(b \rightarrow s \gamma)^{\exp }}{\mathrm{BR}(b \rightarrow s \gamma)^{\mathrm{SM}}}=1.089 \pm 0.27, \\
& \mathrm{BR}\left(B_{s} \rightarrow \mu^{+} \mu^{-}\right)=(2.9 \pm 1.4) \times 10^{-9}
\end{aligned}
$$

For the $B_{u}$ decay to $\tau \nu$ we use $[97,106-108]$ and for $\Delta M_{B_{S}}$ we use $[109,110]$ :

$$
\begin{aligned}
& \frac{\mathrm{BR}\left(B_{u} \rightarrow \tau \nu\right)^{\exp }}{\mathrm{BR}\left(B_{u} \rightarrow \tau \nu\right)^{\mathrm{SM}}}=1.39 \pm 0.69, \\
& \frac{\Delta M_{B_{s}}^{\exp }}{\Delta M_{B_{s}}^{\mathrm{SM}}}=0.97 \pm 0.2 .
\end{aligned}
$$

Finally, we consider Cold Dark Matter (CDM) constraints. Since the Lightest SUSY Particle (LSP), which in our case is the lightest neutralino, is a promising CDM candidate [111, 112], we examine if each model is within the CDM relic density experimental limits. The current bound on the CDM relic density at $2 \sigma$ level is given by [113]

$\Omega_{\mathrm{CDM}} h^{2}=0.1120 \pm 0.0112$.

In the following sections we will apply these constraints to each model and discuss the corresponding collider phenomenology.

\section{Computational setup}

The setup for our phenomenological analysis is as follows. Starting from an appropriate set of MSSM boundary conditions at the GUT scale, parameters are run down to the SUSY scale using a private code. Two-loop RGEs are used throughout, with the exception of the soft sector, in which one-loop RGEs are used. The running parameters are then used as inputs for both FeynHiggs [34-43] and a SARAH [114] generated, custom MSSM module for SPheno [115,116]. It should be noted that FeynHiggs requires the $m_{b}\left(m_{b}\right)$ scale, the physical top quark mass $m_{t}$ as well as the physical pseudoscalar boson mass $M_{A}$ as input. The first two values are calculated by the private code while $M_{A}$ is calculated only in $\overline{\mathrm{DR}}$ scheme. This single value is obtained from the SPheno output where it is calculated at the two-loop level in the gaugeless limit $[117,118]$. The flow of information between codes in our analysis is summarised in Fig. 1.

At this point both codes contain a consistent set of all required parameters. SM-like Higgs boson mass as well as low energy observables mentioned in Sect. 3 are evaluated using FeynHiggs. To obtain collider predictions we use SARAH to generate UFO $[119,120]$ model for MadGraph event generator. Based on SLHA spectrum files generated by SPheno, we use MadGraph5_aMC@NLO [121] to calculate cross sections for Higgs boson and SUSY particle production at the HL-LHC and a $100 \mathrm{TeV}$ FCChh. Processes are generated at the leading order, using NNPDF31_1o_as_0130 [122] structure functions interfaced through LHAPDF 6 [123]. Cross sections are computed using dynamic scale choice, where the scale is set equal to the transverse mass of an event, in 4 or 5 -flavor scheme depending on the presence or not of $b$-quarks in the final state. The results are given in Sects. 5-7.

\section{The minimal $N=1$ supersymmetric $S U$ (5) model}

We start with the partial reduction of the $N=1$ SUSY $S U(5)$ model $[17,48]$. Our notation is as follows: $\Psi^{I}(\mathbf{1 0})$ and $\Phi^{I}(\overline{\mathbf{5}})$ refer to the three generations of leptons and quarks $(I=1,2,3), \Sigma(\mathbf{2 4})$ is the adjoint which breaks $S U(5)$ to $S U(3)_{\mathrm{C}} \times S U(2)_{\mathrm{L}} \times U(1)_{\mathrm{Y}}$ and $\bar{H}(\overline{\mathbf{5}})$ represent the two Higgs superfields for the electroweak symmetry breaking (ESB) [124,125]. The choice of using only one set of $(\mathbf{5}+\overline{\mathbf{5}})$ for the ESB renders the model asymptotically free (i.e. $\beta_{g}<0$ ). The superpotential of the model is described by

$$
\begin{aligned}
W= & \frac{g_{t}}{4} \epsilon^{\alpha \beta \gamma \delta \tau} \Psi_{\alpha \beta}^{(3)} \Psi_{\gamma \delta}^{(3)} H_{\tau}+\sqrt{2} g_{b} \Phi^{(3) \alpha} \Psi_{\alpha \beta}^{(3)} \bar{H}^{\beta} \\
& +\frac{g_{\lambda}}{3} \Sigma_{\alpha}^{\beta} \Sigma_{\beta}^{\gamma} \Sigma_{\gamma}^{\alpha}+g_{f} \bar{H}^{\alpha} \Sigma_{\alpha}^{\beta} H_{\beta} \\
& +\frac{\mu_{\Sigma}}{2} \Sigma_{\alpha}^{\gamma} \Sigma_{\gamma}^{\alpha}+\mu_{H} \bar{H}^{\alpha} H_{\alpha}
\end{aligned}
$$

where only the third generation Yukawa couplings are taken into account. The indices $\alpha, \beta, \gamma, \delta, \tau$ are $S U(5)$ ones. A detailed presentation of the model can be found in [17] as well as in [126,127].

Our primary coupling is the gauge coupling $g$. In this model the gauge-Yukawa unification can be achieved through two sets of solutions which are asymptotically free [17]: 


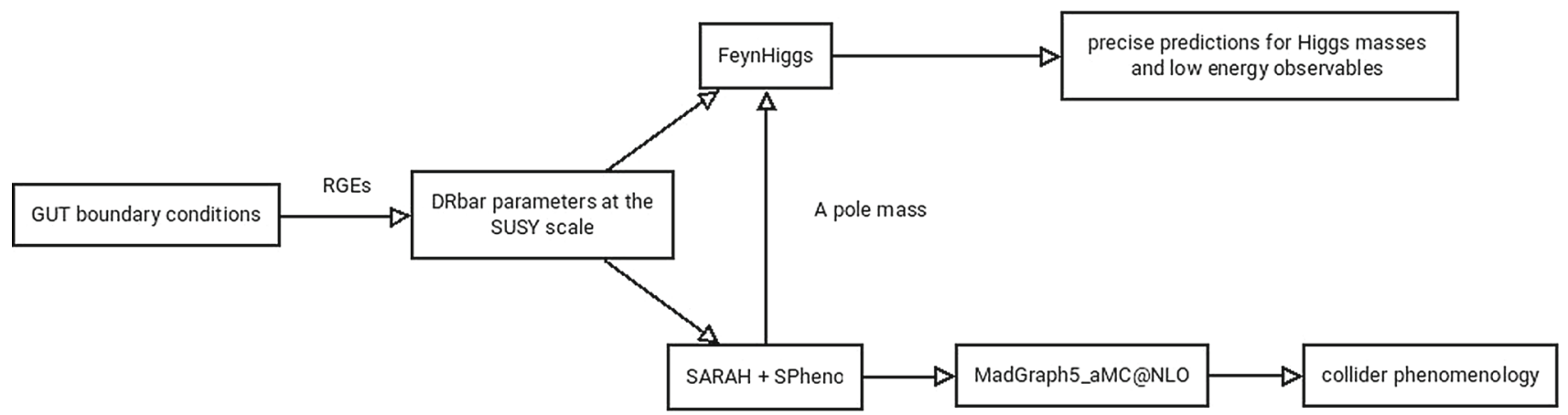

Fig. 1 Flow of information between used computer codes (see text for details)

$a: g_{t}=\sqrt{\frac{2533}{2605}} g+\mathcal{O}\left(g^{3}\right), \quad g_{b}=\sqrt{\frac{1491}{2605}} g+\mathcal{O}\left(g^{3}\right)$,

$g_{\lambda}=0, \quad g_{f}=\sqrt{\frac{560}{521}} g+\mathcal{O}\left(g^{3}\right)$,

$b: g_{t}=\sqrt{\frac{89}{65}} g+\mathcal{O}\left(g^{3}\right)$,

$g_{b}=\sqrt{\frac{63}{65}} g+\mathcal{O}\left(g^{3}\right), \quad g_{\lambda}=0, \quad g_{f}=0$,

where the higher order terms denote uniquely computable power series in $g$. Let us note that the reduction of the dimensionless sector is independent of the dimensionful one. These solutions describe the boundaries of a RGI surface in the parameter space which is $\mathrm{AF}$ and where $g_{f}$ and $g_{\lambda}$ could be different from zero. Therefore, a partial reduction is possible where $g_{\lambda}$ and $g_{f}$ are independent (non-vanishing) parameters without endangering asymptotic freedom (AF). The proton decay constraints favor solution $a$, therefore we choose this one for our discussion. ${ }^{3}$

The SSB Lagrangian is

$$
\begin{aligned}
-\mathcal{L}_{\text {soft }}= & m_{H_{u}}^{2} \hat{H}^{* \alpha} \hat{H}_{\alpha}+m_{H_{d}}^{2} \hat{\bar{H}}_{\alpha}^{*} \hat{\bar{H}}^{\alpha}+m_{\Sigma}^{2} \hat{\Sigma}_{\beta}^{\dagger \alpha} \hat{\Sigma}_{\alpha}^{\beta} \\
& +\sum_{I=1,2,3}\left[m_{\Phi^{I}}^{2} \hat{\Phi}_{\alpha}^{*(I)} \hat{\Phi}^{(I) \alpha}+m_{\Psi^{I}}^{2} \hat{\Psi}^{\dagger(I) \alpha \beta} \hat{\Psi}_{\beta \alpha}^{(I)}\right] \\
& +\left\{\frac{1}{2} M \lambda \lambda+B_{H} \hat{\bar{H}}^{\alpha} \hat{H}_{\alpha}+B_{\Sigma} \hat{\Sigma}_{\beta}^{\alpha} \hat{\Sigma}_{\alpha}^{\beta}\right. \\
& +h_{f} \hat{\bar{H}}^{\alpha} \hat{\Sigma}_{\alpha}^{\beta} \hat{H}_{\beta} \\
& +\frac{h_{\lambda}}{3} \hat{\Sigma}_{\alpha}^{\beta} \hat{\Sigma}_{\beta}^{\gamma} \hat{\Sigma}_{\gamma}^{\alpha}+\frac{h_{t}}{4} \epsilon^{\alpha \beta \gamma \delta \tau} \hat{\Psi}_{\alpha \beta}^{(3)} \hat{\Psi}_{\gamma \delta}^{(3)} \hat{H}_{\tau} \\
& \left.+\sqrt{2} h_{b} \hat{\Phi}^{(3) \alpha} \hat{\Psi}_{\alpha \beta}^{(3)} \hat{\bar{H}}^{\beta}+\text { h.c. }\right\},
\end{aligned}
$$

where the hat denotes the scalar components of the chiral superfields. The parameters $M, \mu_{\Sigma}$ and $\mu_{H}$ are treated as

$3 g_{\lambda}=0$ is inconsistent, but $g_{\lambda} \lesssim 0.005$ is necessary in order for the proton decay constraint [21] to be satisfied. A small $g_{\lambda}$ is expected to not affect the prediction of unification of SSB parameters. independent ones, since they cannot be reduced in a suitable form. The lowest-order reduction for the parameters of the SSB Lagrangian are given by:

$B_{H}=\frac{1029}{521} \mu_{H} M, \quad B_{\Sigma}=-\frac{3100}{521} \mu_{\Sigma} M$,

$h_{t}=-g_{t} M, \quad h_{b}=-g_{b} M, \quad h_{f}=-g_{f} M, \quad h_{\lambda}=0$,

$m_{H_{u}}^{2}=-\frac{569}{521} M^{2}, \quad m_{H_{d}}^{2}=-\frac{460}{521} M^{2}, \quad m_{\Sigma}^{2}=\frac{1550}{521} M^{2}$,

$m_{\Phi^{3}}^{2}=\frac{436}{521} M^{2}, \quad m_{\Phi^{1,2}}^{2}=\frac{8}{5} M^{2}$,

$m_{\Psi^{3}}^{2}=\frac{545}{521} M^{2}, \quad m_{\Psi^{1,2}}^{2}=\frac{12}{5} M^{2}$.

We choose the gaugino mass $M$ for characterizing the SUSY breaking scale. Finally, we note that (i) $B_{\Sigma}$ and $B_{H}$ are treated as independent parameters without spoiling the oneloop reduction solution of Eq. (35) and (ii) the soft scalar mass sum rule still holds despite the specific relations among the gaugino mass and the soft scalar masses.

We analyze the particle spectrum predicted for $\mu<0$ as the only phenomenologically acceptable choice (in the $\mu>0$ the quark masses do not match the experimental measurements). Below $M_{\mathrm{GUT}}$ all couplings and masses of the theory run according to the RGEs of the MSSM. Thus we examine the evolution of these parameters according to their RGEs up to two-loops for dimensionless parameters and at one-loop for dimensionful ones imposing the corresponding boundary conditions.

As presented in [44], the pole top mass $m_{t}$ is predicted within $2 \sigma$ of Eq. (25). Concerning the $m_{b}\left(M_{Z}\right)$ prediction (also in [44]), we take into account a theoretical uncertainty of $\sim 3 \%$. But even taking theoretical and experimental uncertainties into account in combination, we find agreement with the experimental value only at the $4 \sigma$ level. However, since there additional uncertainties of a few percent on the quark Yukawa couplings at the SUSY-breaking scale, that were not fully included (see [21]) into the evaluation of the bottom mass, we still consider the model as viable and proceed with its analysis. 



Fig. 2 Scatter plots for the minimal $N=1 S U(5)$ model. Left: the lightest Higgs mass, $M_{h}$, as a function of $M$. The $B$-physics constraints allow (mostly) higher scale points (with green color). Right: the theoretical uncertainty of the light Higgs mass [43]

The prediction for $M_{h}$ as a function of the unified gaugino mass $M$ with $\mu<0$ is given in Fig. 2 (left). The $\Delta M_{B_{s}}$ channel is responsible for the gap at the $B$-physics allowed points (green points). The scattered points come from the fact that for each $M$ we vary the free parameters $\mu_{\Sigma}$ and $\mu_{H}$. Figure 2 (right) gives the theoretical uncertainty of the Higgs mass for each point, calculated with FeynHiggs 2.16.0 [43]. There is substantial improvement to the Higgs mass uncertainty compared to past analyses, since it has dropped by more than $1 \mathrm{GeV}$.

Large parts of the predicted particle spectrum are in agreement with the $B$-physics observables and the lightest Higgs boson mass measurement and its theoretical uncertainty. We choose three benchmarks in the low-mass region, marking the points with the lightest SUSY particle (LSP) above $1200 \mathrm{GeV}$ (MINI-1), $1500 \mathrm{GeV}$ (MINI-2) and $2200 \mathrm{GeV}$ (MINI-3), respectively. The mass of the LSP can go as high as $\sim 3800 \mathrm{GeV}$, but the cross sections calculated below will then be negligible and we restrict ourselves here to the low-mass region. The values presented in Table 1 were used as input to get the full supersymmetric spectrum from SPheno 4.0.4 [115,116]. $M_{i}$ are the gaugino masses and the rest are squared soft sfermion masses which are diagonal $\left(\mathbf{m}^{2}=\operatorname{diag}\left(\mathrm{m}_{1}^{2}, \mathrm{~m}_{2}^{2}, \mathrm{~m}_{3}^{2}\right)\right)$, and soft trilinear couplings (also diagonal $\mathbf{A}_{\mathbf{i}}=\mathbb{1}_{3 \times 3} A_{i}$ ).

The resulting masses of all the particles that will be relevant for our analysis can be found in Table 2. The three first values are the heavy Higgs masses. The gluino mass is $M_{\tilde{g}}$, the neutralinos and the charginos are denoted as $M_{\tilde{\chi}_{i}^{0}}$ and $M_{\tilde{\chi}_{i}^{ \pm}}$, while the slepton and sneutrino masses for all three generations are given as $M_{\tilde{e}_{1,2,3}}, M_{\tilde{v}_{1,2,3}}$. Similarly, the squarks are denoted as $M_{\tilde{d}_{1,2}}$ and $M_{\tilde{u}_{1,2}}$ for the first two generations. The third generation masses are given by $M_{\tilde{t}_{1,2}}$ for stops and $M_{\tilde{b}_{1,2}}$ for sbottoms.

At this point there is an important remark. No point fulfills the strict bound of Eq. (30), since we have overproduction of CDM in the early universe. The LSP, which in our case is the lightest neutralino, is strongly Bino-like. Combined with the heavy mass it acquires (1-2 TeV), it cannot account for a relic density low enough to agree with experimental observation. Thus, we need a mechanism that reduces this CDM abundance. This could be related to the problem of neutrino masses, which cannot be generated naturally in this particular model. However, one could extend the model by considering bilinear R-parity violating terms and thus introduce neutrino masses $[128,129]$. Rparity violation [130-133] would have a small impact on the collider phenomenology, but remove the CDM bound of Eq. (30) completely. Other mechanisms, not involving Rparity violation, that could be invoked if the amount of CDM appears to be too large, concern the cosmology of the early universe. For example, "thermal inflation" [134] or "late time entropy injection" [135] can bring the CDM density into agreement with Planck measurements. For the original discussion see [44].

Table 3 shows the expected production cross section for selected channels at the $100 \mathrm{TeV}$ future FCC-hh collider. We do not show any cross sections for $\sqrt{s}=14 \mathrm{TeV}$, since the prospects for discovery of MINI scenarios at the HL-LHC are very dim. SUSY particles are too heavy to be produced with cross sections greater that $0.01 \mathrm{fb}$. Concerning the heavy Higgs bosons, the main search channels will be $H / A \rightarrow$ $\tau^{+} \tau^{-}$. Our heavy Higgs-boson mass scale shows values $\gtrsim$ $2500 \mathrm{GeV}$ with $\tan \beta \sim 50$. The corresponding reach of the HL-LHC has been estimated in $[136,137]$. In comparison with our benchmark points we conclude that they will not be accessible at the HL-LHC. ${ }^{4}$

The situation changes for the FCC-hh [138]. Theory analyses $[139,140]$ have shown that for large $\tan \beta$ heavy Higgsboson mass scales up to $\sim 8 \mathrm{TeV}$ may be accessible, both for neutral as well as for charged Higgs bosons. The relevant

\footnotetext{
$\overline{4}$ The analysis presented in $[136,137]$ only reaches $M_{A} \leq 2000 \mathrm{GeV}$, where an exclusion down to $\tan \beta \sim 30$ is expected. An extrapolation to $\tan \beta \sim 50$ reaches Higgs-boson mass scales of $\sim 2500 \mathrm{GeV}$.
} 
Table 1 Minimal $N=1 S U(5)$ predictions that are used as input to SPheno. Mass parameters are in $\mathrm{GeV}$ and rounded to $1 \mathrm{GeV}$

\begin{tabular}{lllllllllll}
\hline & $M_{1}$ & $M_{2}$ & $M_{3}$ & $|\mu|$ & $b$ & $A_{u}$ & $A_{d}$ & $A_{e}$ & $\tan \beta$ & $m_{Q_{1,2}}^{2}$ \\
\hline MINI-1 & 1227 & 2228 & 5310 & 4236 & $401^{2}$ & 4325 & 4772 & 1732 & 50.3 & $6171^{2}$ \\
MINI-2 & 1507 & 2721 & 6376 & 5091 & $496^{2}$ & 5245 & 5586 & 2005 & 52.0 & $7445^{2}$ \\
MINI-3 & 2249 & 4019 & 9138 & 7367 & $1246^{2}$ & 7571 & 8317 & 3271 & 50.3 & $10762^{2}$ \\
\hline & $m_{Q_{3}}^{2}$ & $m_{L_{1,2}}^{2}$ & $m_{L_{3}}^{2}$ & $m_{\bar{u}_{1,2}}^{2}$ & $m_{\bar{u}_{3}}^{2}$ & $m_{\bar{d}_{1,2}}^{2}$ & $m_{\bar{d}_{3}}^{2}$ & $m_{\bar{e}_{1,2}}^{2}$ & $m_{\bar{e}_{3}}^{2}$ \\
\hline MINI-1 & $4548^{2}$ & $3714^{2}$ & $2767^{2}$ & $5974^{2}$ & $4181^{2}$ & $5478^{2}$ & $4177^{2}$ & $4160^{2}$ & $2491^{2}$ \\
MINI-2 & $5469^{2}$ & $4521^{2}$ & $3358^{2}$ & $7206^{2}$ & $5039^{2}$ & $5478^{2}$ & $4994^{2}$ & $5070^{2}$ & $3019^{2}$ \\
MINI-3 & $7890^{2}$ & $6639^{2}$ & $4934^{2}$ & $10412^{2}$ & $7233^{2}$ & $9495^{2}$ & $7211^{2}$ & $7459^{2}$ & $4464^{2}$
\end{tabular}

Table 2 Masses of Higgs bosons and some of the SUSY particles for each benchmark of the Minimal $N=1 S U(5)$ (in TeV)

\begin{tabular}{lllllllllll}
\hline & $M_{H}$ & $M_{A}$ & $M_{H^{ \pm}}$ & $M_{\tilde{g}}$ & $M_{\tilde{\chi}_{1}^{0}}$ & $M_{\tilde{\chi}_{2}^{0}}$ & $M_{\tilde{\chi}_{3}^{0}}$ & $M_{\tilde{\chi}_{4}^{0}}$ & $M_{\tilde{\chi}_{1}^{ \pm}}$ & $M_{\tilde{\chi}_{2}^{ \pm}}$ \\
\hline MINI-1 & 2.660 & 2.660 & 2.637 & 5.596 & 1.221 & 2.316 & 4.224 & 4.225 & 2.316 & 4.225 \\
MINI-2 & 3.329 & 3.329 & 3.300 & 6.717 & 1.500 & 2.827 & 5.076 & 5.077 & 2.827 & 5.078 \\
MINI-3 & 8.656 & 8.656 & 8.631 & 9.618 & 2.239 & 4.176 & 7.357 & 7.358 & 4.176 & 7.359 \\
\hline & $M_{\tilde{e}_{1,2}}$ & $M_{\tilde{\nu}_{1,2}}$ & $M_{\tilde{\tau}}$ & $M_{\tilde{\nu}_{\tau}}$ & $M_{\tilde{d}_{1,2}}$ & $M_{\tilde{u}_{1,2}}$ & $M_{\tilde{b}_{1}}$ & $M_{\tilde{b}_{2}}$ & $M_{\tilde{t}_{1}}$ & $M_{\tilde{t}_{2}}$ \\
\hline MINI-1 & 3.729 & 3.728 & 2.445 & 2.766 & 5.617 & 6.100 & 4.332 & 4.698 & 4.312 & 4.704 \\
MINI-2 & 4.539 & 4.538 & 2.968 & 3.356 & 6.759 & 7.354 & 5.180 & 5.647 & 5.197 & 5.652 \\
MINI-3 & 6.666 & 6.665 & 4.408 & 4.935 & 9.722 & 10.616 & 7.471 & 8.148 & 7.477 & 8.151 \\
\hline
\end{tabular}

Table 3 Expected production cross sections (in fb) for SUSY particles in the MINI scenarios. There are no channels with cross sections exceeding $0.01 \mathrm{fb}$ at $\sqrt{s}=14 \mathrm{TeV}$

\begin{tabular}{|c|c|c|c|c|c|c|c|}
\hline Scenarios $\sqrt{s}$ & MINI-1 $100 \mathrm{TeV}$ & MINI-2 $100 \mathrm{TeV}$ & MINI-3 $100 \mathrm{TeV}$ & Scenarios $\sqrt{s}$ & MINI-1 $100 \mathrm{TeV}$ & MINI-2 $100 \mathrm{TeV}$ & MINI-3 $100 \mathrm{TeV}$ \\
\hline$\tilde{\chi}_{1}^{0} \tilde{\chi}_{1}^{0}$ & 0.04 & 0.02 & & $\tilde{u}_{i} \tilde{\chi}_{1}^{-}, \tilde{d}_{i} \tilde{\chi}_{1}^{+}+$h.c. & 1.00 & 0.35 & 0.03 \\
\hline$\tilde{\chi}_{1}^{0} \tilde{\chi}_{3}^{0}$ & 0.02 & 0.01 & & $\tilde{u}_{i} \tilde{\chi}_{2}^{-}, \tilde{d}_{i} \tilde{\chi}_{2}^{+}+$h.c. & 0.07 & 0.02 & \\
\hline$\tilde{\chi}_{2}^{0} \tilde{\chi}_{2}^{0}$ & 0.06 & 0.02 & & $\tilde{q}_{i} \tilde{\chi}_{1}^{0}, \tilde{q}_{i}^{*} \tilde{\chi}_{1}^{0}$ & 0.38 & 0.14 & 0.02 \\
\hline$\tilde{\chi}_{2}^{0} \tilde{\chi}_{3}^{0}$ & 0.03 & 0.01 & & $\tilde{q}_{i} \tilde{\chi}_{2}^{0}, \tilde{q}_{i}^{*} \tilde{\chi}_{2}^{0}$ & 0.51 & 0.17 & 0.02 \\
\hline$\tilde{\chi}_{2}^{0} \tilde{\chi}_{4}^{0}$ & 0.02 & 0.01 & & $\tilde{v}_{i} \tilde{e}_{j}^{*}, \tilde{v}_{i}^{*} \tilde{e}_{j}$ & 0.06 & 0.02 & \\
\hline$\tilde{\chi}_{3}^{0} \tilde{\chi}_{4}^{0}$ & 0.05 & 0.02 & & $H b \bar{b}$ & 84.04 & 30.10 & 0.17 \\
\hline$\tilde{\chi}_{2}^{0} \tilde{\chi}_{1}^{+}$ & 2.20 & 0.98 & 0.18 & $A b \bar{b}$ & 84.79 & 29.79 & 0.18 \\
\hline$\tilde{\chi}_{3}^{0} \tilde{\chi}_{2}^{+}$ & 0.10 & 0.04 & 0.01 & $H^{+} b \bar{t}+H^{-} t \bar{b}$ & 33.24 & 12.76 & 0.1 \\
\hline$\tilde{\chi}_{4}^{0} \tilde{\chi}_{2}^{+}$ & 0.10 & 0.04 & 0.01 & $H^{-} b \bar{b}$ & 0.04 & 0.02 & \\
\hline$\tilde{g} \tilde{g}$ & 7.76 & 2.02 & 0.11 & $H t \bar{t}$ & 0.03 & 0.01 & \\
\hline$\tilde{g} \tilde{\chi}_{1}^{0}$ & 0.28 & 0.11 & 0.01 & $A t \bar{t}$ & 0.02 & 0.01 & \\
\hline$\tilde{g} \tilde{\chi}_{2}^{0}$ & 0.34 & 0.12 & 0.01 & $H t b$ & 0.01 & & \\
\hline$\tilde{g} \tilde{\chi}_{1}^{+}$ & 0.70 & 0.27 & 0.03 & $H A$ & 0.03 & 0.01 & \\
\hline$\tilde{q}_{i} \tilde{q}_{j}, \tilde{q}_{i} \tilde{q}_{j}^{*}$ & 21.15 & 7.44 & 0.74 & $H H^{+}$ & 0.06 & 0.02 & \\
\hline$\tilde{\chi}_{1}^{+} \tilde{\chi}_{1}^{-}$ & 1.19 & 0.54 & 0.09 & $H^{+} W^{-}$ & 6.50 & 2.96 & 0.03 \\
\hline$\tilde{\chi}_{1}^{+} \tilde{\chi}_{2}^{-}$ & 0.05 & 0.02 & & $H W^{+}$ & 0.02 & 0.01 & \\
\hline$\tilde{\chi}_{2}^{+} \tilde{\chi}_{1}^{-}$ & 0.05 & 0.02 & & $H^{+} H^{-}$ & 0.04 & 0.01 & \\
\hline$\tilde{\chi}_{2}^{+} \tilde{\chi}_{2}^{-}$ & 0.06 & 0.02 & & $A H^{+}$ & 0.06 & 0.02 & \\
\hline$\tilde{e}_{i} \tilde{e}_{j}^{*}$ & 0.16 & 0.08 & 0.01 & $A W^{+}$ & 0.02 & 0.01 & \\
\hline$\tilde{q}_{i} \tilde{g}, \tilde{q}_{i}^{*} \tilde{g}$ & 30.57 & 9.33 & 0.66 & $H Z$ & 1.38 & 0.58 & 0.01 \\
\hline$\tilde{v}_{i} \tilde{v}_{j}^{*}$ & 0.04 & 0.02 & & $A Z$ & 1.20 & 0.52 & 0.01 \\
\hline
\end{tabular}


decay channels are $H / A \rightarrow \tau^{+} \tau^{-}$and $H^{ \pm} \rightarrow \tau \nu_{\tau}$, tb. This places our three benchmark points well within the covered region (MINI-1 and MINI-2) or at the border of the parameter space that can be probed (MINI-3).

The energy of $100 \mathrm{TeV}$ is big enough to produce SUSY particles in pairs. However, the cross sections remain relatively small. Only for the MINI- 1 scenario the squark pair and squark-gluino (summed over all squarks) production cross sections can reach tens of fb. For MINI-2 and MINI-3 scenarios the cross sections are significantly smaller. In these scenarios squarks decay preferentially into a quark+LSP (with $\mathrm{BR} \sim 0.95)$, gluino into $\tilde{t} \bar{t}$ and $\tilde{b} \bar{b}+h . c$ with $\mathrm{BR} \sim 0.33$ each.

The SUSY discovery reach at the FCC-hh with $3 \mathrm{ab}^{-1}$ was evaluated in [141] for a certain set of simplified models. In the following we will compare these simplified model limits with our benchmark points to get an idea, which part of the spectrum can be covered at the FCC-hh. A more detailed evaluation with the future limits implemented into proper recasting tools would be necessary to obtain a firmer statement. However, such a detailed analysis goes beyond the scope of our paper and we restrict ourselves to the simpler direct comparison of the simplified model limits with our benchmark predictions.

Concerning the scalar tops, the mass predictions of MINI1 and MINI-2 are well within the anticipated reach of the FCC-hh, while MINI-3 predicts a too heavy stop mass. On the other hand, even for MINI- 1 and MINI- 2 no $5 \sigma$ discovery can be expected. The situation looks more favorable for the first and second generation squarks. All the predicted masses can be excluded at the FCC-hh, whereas a $5 \sigma$ discovery will be difficult, but potentially possible (see Fig. 19 in [141]). Even more favorable appear the prospects for gluino searches at the FCC-hh. All three benchmark points may lead to a $5 \sigma$ discovery (see Fig. 13 in [141]). On the other hand, chances for chargino/neutralino searches are slim at the FCC-hh. The Next-to LSP (NLSP) can only be accessed for $M_{\tilde{\chi}_{1}^{0}} \lesssim 1 \mathrm{TeV}$ (see Fig. 21 in [141]), where all our benchmark points have $M_{\tilde{\chi}_{1}^{0}}>1 \mathrm{TeV}$. Taking into account that our three benchmark points represent only the lower part of the possible mass spectrum (with LSP masses of up to $\sim 1.5 \mathrm{TeV}$ higher), we conclude that even at the FCC-hh large parts of the possible SUSY spectrum will remain elusive.

\section{The finite $N=1$ supersymmetric $S U(5)$ model}

We proceed now to the finite to all-orders $S U(5)$ gauge theory, where the reduction of couplings is restricted to the third generation. An older examination of this specific finite unified theory (FUT) was shown to be in agreement with the experimental constraints at the time [30] and has predicted, almost five years before its discovery, the light Higgs mass in the correct range. As discussed below, improved Higgs calculations predict a somewhat different interval that is still in agreement with current experimental data. The particle content of the model has three $(\overline{\mathbf{5}}+\mathbf{1 0})$ supermultiplets for the three generations of leptons and quarks, while the Higgs sector consists of four supermultiplets $(\overline{\mathbf{5}}+\mathbf{5})$ and one 24. The finite $S U$ (5) group is broken to the MSSM, which of course in no longer a finite theory [15-18,22,25].

In order for this finite to all-orders $S U$ (5) model to achieve gauge Yukawa unification (GYU), it should have the following characteristics:

(i) The one-loop anomalous dimensions are diagonal i.e., $\gamma_{i}^{(1) j} \propto \delta_{i}^{j}$.

(ii) The fermions of the $\overline{\mathbf{5}}_{i}$ and $\mathbf{1 0}_{i}(i=1,2,3)$ are not coupled to the 24 .

(iii) The pair of the MSSM Higgs doublets are mostly composed from the 5 and $\overline{5}$ Higgs that couple to the third generation

The superpotential of the model, with an enhanced symmetry due to the reduction of couplings, is given by $[26,28]$ :

$$
\begin{aligned}
W= & \sum_{i=1}^{3}\left[\frac{1}{2} g_{i}^{u} \mathbf{1 0}_{i} \mathbf{1 0}_{i} H_{i}+g_{i}^{d} \mathbf{1 0}_{i} \overline{\mathbf{5}}_{i} \bar{H}_{i}\right] \\
& +g_{23}^{u} \mathbf{1 0}_{2} \mathbf{1 0}_{3} H_{4} \\
& +g_{23}^{d} \mathbf{1 0}_{2} \overline{\mathbf{5}}_{3} \bar{H}_{4}+g_{32}^{d} \mathbf{1 0}_{3} \overline{\mathbf{5}}_{2} \bar{H}_{4}+g_{2}^{f} H_{2} \mathbf{2 4} \bar{H}_{2} \\
& +g_{3}^{f} H_{3} \mathbf{2 4} \bar{H}_{3}+\frac{g^{\lambda}}{3}(\mathbf{2 4})^{3} .
\end{aligned}
$$

Discussion of the model with a more detailed description can be found in [15-17]. The non-degenerate and isolated solutions to the vanishing of $\gamma_{i}^{(1)}$ are:

$$
\begin{aligned}
& \left(g_{1}^{u}\right)^{2}=\frac{8}{5} g^{2}, \quad\left(g_{1}^{d}\right)^{2}=\frac{6}{5} g^{2}, \\
& \left(g_{2}^{u}\right)^{2}=\left(g_{3}^{u}\right)^{2}=\frac{4}{5} g^{2}, \\
& \left(g_{2}^{d}\right)^{2}=\left(g_{3}^{d}\right)^{2}=\frac{3}{5} g^{2}, \quad\left(g_{23}^{u}\right)^{2}=\frac{4}{5} g^{2}, \\
& \left(g_{23}^{d}\right)^{2}=\left(g_{32}^{d}\right)^{2}=\frac{3}{5} g^{2}, \\
& \left(g^{\lambda}\right)^{2}=\frac{15}{7} g^{2}, \quad\left(g_{2}^{f}\right)^{2}=\left(g_{3}^{f}\right)^{2}=\frac{1}{2} g^{2}, \\
& \left(g_{1}^{f}\right)^{2}=0, \quad\left(g_{4}^{f}\right)^{2}=0 .
\end{aligned}
$$



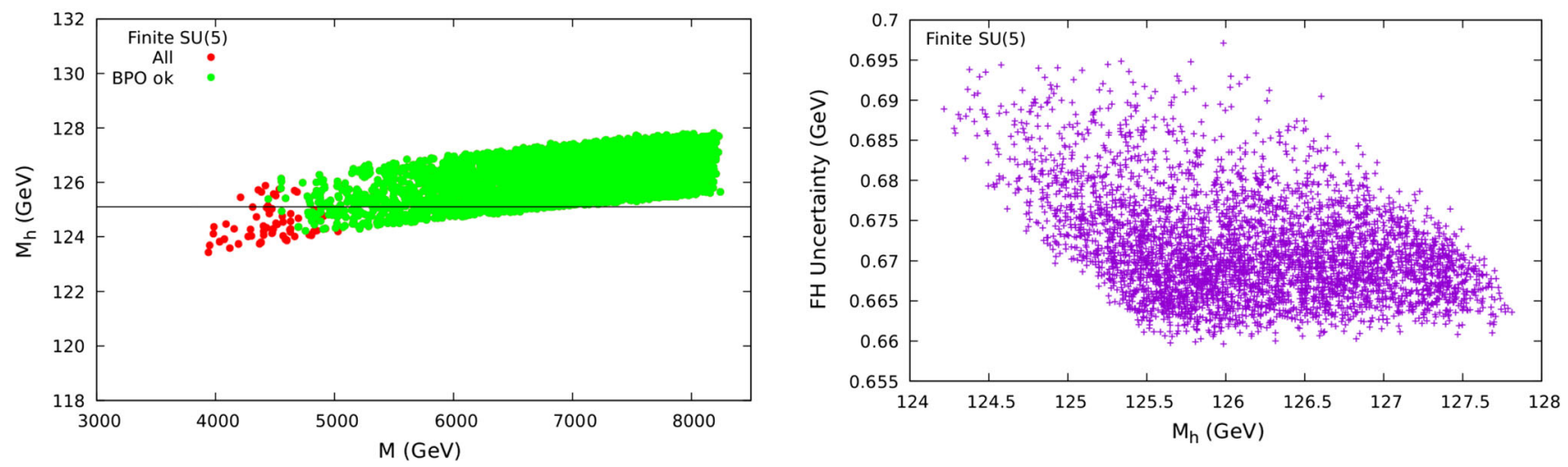

Fig. 3 Scatter plot for the finite $N=1 S U(5)$ model. Left: $M_{h}$ as a function of $M$. Green points comply with $B$-physics constraints. Right: the lightest Higgs mass theoretical uncertainty calculated with FeynHiggs 2.16.0 [43]

We have also the relation $h=-M C$, while the sum rules lead to:

$m_{H_{u}}^{2}+2 m_{\mathbf{1 0}}^{2}=M^{2}, \quad m_{H_{d}}^{2}-2 m_{\mathbf{1 0}}^{2}=-\frac{M^{2}}{3}$,

$m_{\overline{5}}^{2}+3 m_{10}^{2}=\frac{4 M^{2}}{3}$.

Therefore, we only have two free parameters, namely $m_{10}$ and $M$ in the dimensionful sector.

When $S U(5)$ breaks down to the MSSM, a suitable rotation in the Higgs sector [15,16,142-145], permits only a pair of Higgs doublets (coupled mostly to the third family) to remain light and acquire vev's. Avoiding fast proton decay is achieved with the usual doublet-triplet splitting, although different from the one applied to the minimal $S U(5)$ due to the extended Higgs sector of the finite model. Therefore, below the GUT scale we get the MSSM where the third generation is given by the finiteness conditions while the first two remain unrestricted.

Conditions set by finiteness do not restrict the renormalization properties at low energies, so we are left with boundary conditions on the gauge and Yukawa couplings (37), the $h=-M C$ relation and the soft scalar-mass sum rule at $M_{\mathrm{GUT}}$. The quark masses $m_{b}\left(M_{Z}\right)$ and $m_{t}$ are predicted within $2 \sigma$ and $3 \sigma$ uncertainty, respectively of their experimental values (see [44] for details). The only phenomenologically viable option is to consider $\mu<0$, as shown in [44, 146-152].

The scatter plot of the light Higgs boson mass is given in Fig. 3 (left), while its theory uncertainty [43] is given in Fig. 3 (right), with the same color coding as in Fig. 2. This point-by-point uncertainty (calculated with FeynHiggs) drops significantly (w.r.t. past analyses) to $0.65-0.70 \mathrm{GeV}$. The scattered points come from the free parameter $m_{10}$.

Compared to our previous analyses [44,146-156], the improved evaluation of $M_{h}$ and its uncertainty prefer a heavier (Higgs) spectrum and thus allows only a heavy super- symmetric spectrum (which is in agreement with all existing experimental constraints). In particular, very heavy colored SUSY particles are favored (nearly independent of the $M_{h}$ uncertainty), in agreement with the non-observation of those particles at the LHC [157].

We choose three benchmarks, each featuring the LSP above $2100 \mathrm{GeV}, 2400 \mathrm{GeV}$ and $2900 \mathrm{GeV}$ respectively. Again, they are chosen from the low-mass region. Although the LSP can be as heavy as $\sim 4000 \mathrm{GeV}$, but in such cases the production cross sections even at the FCC-hh would be too small. The input and output of SPheno 4.0.4 [115,116] can be found in Tables 4 and 5 (with the notation as in Sect. 5).

Concerning DM, the model exhibits a high relic abundance for CDM, as the lightest neutralino (which is the LSP) is again strongly Bino-like (see [44]). The CDM alternatives proposed for the Minimal $S U(5)$ model can also be applied here. It should be noted that the bilinear R-parity violating terms proposed in the previous section preserve finiteness, as well.

The expected production cross sections for various final states are listed in Table 6. At $14 \mathrm{TeV}$ HL-LHC none of the Finite $N=1 S U(5)$ scenarios listed in Table 4 has a SUSY production cross section above $0.01 \mathrm{fb}$, and thus will (likely) remain unobservable. All superpartners are too heavy to be produced in pairs. Also the heavy Higgs bosons are far outside the reach of the HL-LHC $[136,137]$.

At the FCC-hh the discovery prospects for the heavy Higgs-boson spectrum is significantly better. With $\tan \beta \sim$ 50 the first two benchmark points, FUTSU5-1 and FUTSU52 , are well within the reach of the FCC-hh. The third point, FUTSU5-3, however, with $M_{A} \sim 16 \mathrm{TeV}$ will be far outside the reach of the FCC-hh. Prospects for detecting production of squark pairs and squark-gluino pairs are also very dim since their production cross section is also at the level of a few $\mathrm{fb}$. This is as a result of a heavy spectrum in this class of models (see [141] with the same Figures as discussed in Sect. 5). Concerning the stops, the lighter one might be accessible 
Table 4 Finite $N=1 S U(5)$ predictions that are used as input to SPheno. Mass parameters are in GeV and rounded to $1 \mathrm{GeV}$

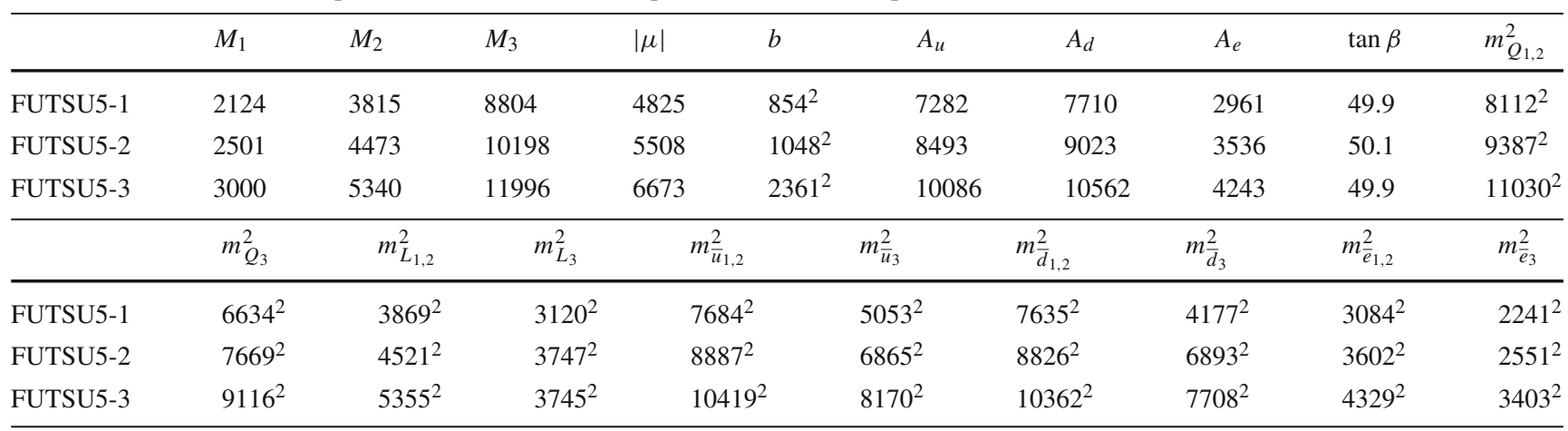

Table 5 Masses for each benchmark of the Finite $N=1 S U(5)$ (in TeV)

\begin{tabular}{|c|c|c|c|c|c|c|c|c|c|c|}
\hline & $M_{H}$ & $M_{A}$ & $M_{H^{ \pm}}$ & $M_{\tilde{g}}$ & $M_{\tilde{\chi}_{1}^{0}}$ & $M_{\tilde{\chi}_{2}^{0}}$ & $M_{\tilde{\chi}_{3}^{0}}$ & $M_{\tilde{\chi}_{4}^{0}}$ & $M_{\tilde{\chi}_{1}^{ \pm}}$ & $M_{\tilde{\chi}_{2}^{ \pm}}$ \\
\hline FUTSU5-1 & 5.688 & 5.688 & 5.688 & 8.966 & 2.103 & 3.917 & 4.829 & 4.832 & 3.917 & 4.833 \\
\hline FUTSU5-2 & 7.039 & 7.039 & 7.086 & 10.380 & 2.476 & 4.592 & 5.515 & 5.518 & 4.592 & 5.519 \\
\hline \multirow[t]{2}{*}{ FUTSU5-3 } & 16.382 & 16.382 & 16.401 & 12.210 & 2.972 & 5.484 & 6.688 & 6.691 & 5.484 & 6.691 \\
\hline & $M_{\tilde{e}_{1,2}}$ & $M_{\tilde{v}_{1,2}}$ & $M_{\tilde{\tau}}$ & $M_{\tilde{v}_{\tau}}$ & $M_{\tilde{d}_{1,2}}$ & $M_{\tilde{u}_{1,2}}$ & $M_{\tilde{b}_{1}}$ & $M_{\tilde{b}_{2}}$ & $M_{\tilde{t}_{1}}$ & $M_{\tilde{t}_{2}}$ \\
\hline FUTSU5-1 & 3.102 & 3.907 & 2.205 & 3.137 & 7.839 & 7.888 & 6.102 & 6.817 & 6.099 & 6.821 \\
\hline FUTSU5-2 & 3.623 & 4.566 & 2.517 & 3.768 & 9.059 & 9.119 & 7.113 & 7.877 & 7.032 & 7.881 \\
\hline FUTSU5-3 & 4.334 & 5.418 & 3.426 & 3.834 & 10.635 & 10.699 & 8.000 & 9.387 & 8.401 & 9.390 \\
\hline
\end{tabular}

in FUTSU5-1. For the squarks of the first two generations the prospects of testing the model are somewhat better. All three benchmark models could possibly be excluded at the $2 \sigma$ level, but no discovery at the $5 \sigma$ can be expected. The same holds for the gluino. Charginos and neutralinos will remain unobservable due to the heavy LSP. As in the previous section, since only the lower part of the possible mass spectrum has been considered (with LSP masses higher by up to $\sim 1 \mathrm{TeV}$ ), we have to conclude that again large parts of the possible mass spectra will not be observable at the FCC-hh.

\section{The finite $S U(N)^{3}$ model}

We proceed now to a FUT based on a product gauge group. Consider an $N=1$ SUSY theory with $S U(N)_{1} \times$ $S U(N)_{2} \times \cdots \times S U(N)_{k}$ having $n_{f}$ families transforming as $\left(N, N^{*}, 1, \ldots, 1\right)+\left(1, N, N^{*}, \ldots, 1\right)+\cdots+\left(N^{*}, 1,1, \ldots, N\right)$. Then, the first order coefficient of the $\beta$-function, for each $S U(N)$ group is:

$b=\left(-\frac{11}{3}+\frac{2}{3}\right) N+n_{f}\left(\frac{2}{3}+\frac{1}{3}\right)\left(\frac{1}{2}\right) 2 N=-3 N+n_{f} N$.
Demanding the vanishing of the gauge one-loop $\beta$-function, i.e. $b=0$, we are led to the choice $n_{f}=3$. Phenomenological reasons lead to the choice of the $S U(3)_{C} \times S U(3)_{L} \times S U(3)_{R}$ model, discussed in Ref. [158], while a detailed discussion of the general well known example can be found in [159-162]. The leptons and quarks transform as:

$q=\left(\begin{array}{lll}d & u & D \\ d & u & D \\ d & u & D\end{array}\right) \sim\left(3,3^{*}, 1\right)$

$q^{c}=\left(\begin{array}{lll}d^{c} & d^{c} & d^{c} \\ u^{c} & u^{c} & u^{c} \\ D^{c} & D^{c} & D^{c}\end{array}\right) \sim\left(3^{*}, 1,3\right)$,

$\lambda=\left(\begin{array}{ccc}N & E^{c} & v \\ E & N^{c} & e \\ v^{c} & e^{c} & S\end{array}\right) \sim\left(1,3,3^{*}\right)$

where $D$ are down-type quarks acquiring masses close to $M_{\mathrm{GUT}}$. A cyclic $Z_{3}$ symmetry is imposed on the multiplets to achieve equal gauge couplings at the GUT scale and in that case the vanishing of the first-order $\beta$-function is satisfied. Continuing to the vanishing of the anomalous dimension of all the fields (see Eq. (20)), we note that there are two trilinear invariant terms in the superpotential, namely: 
Table 6 Expected production cross sections (in fb) for SUSY particles in the FUTSU5 scenarios

\begin{tabular}{|c|c|c|c|c|c|c|c|}
\hline $\begin{array}{l}\text { Scenarios } \\
\sqrt{s}\end{array}$ & $\begin{array}{l}\text { FUTSU5-1 } \\
100 \mathrm{TeV}\end{array}$ & $\begin{array}{l}\text { FUTSU5-2 } \\
100 \mathrm{TeV}\end{array}$ & $\begin{array}{l}\text { FUTSU5-3 } \\
100 \mathrm{TeV}\end{array}$ & $\begin{array}{l}\text { Scenarios } \\
\sqrt{s}\end{array}$ & $\begin{array}{l}\text { FUTSU5-1 } \\
100 \mathrm{TeV}\end{array}$ & $\begin{array}{l}\text { FUTSU5-2 } \\
100 \mathrm{TeV}\end{array}$ & $\begin{array}{l}\text { FUTSU5-3 } \\
100 \mathrm{TeV}\end{array}$ \\
\hline$\tilde{\chi}_{2}^{0} \tilde{\chi}_{3}^{0}$ & 0.01 & 0.01 & & $\tilde{v}_{i} \tilde{v}_{j}^{*}$ & 0.02 & 0.01 & 0.01 \\
\hline$\tilde{\chi}_{3}^{0} \tilde{\chi}_{4}^{0}$ & 0.03 & 0.01 & & $\tilde{u}_{i} \tilde{\chi}_{1}^{-}, \tilde{d}_{i} \tilde{\chi}_{1}^{+}+$h.c. & 0.15 & 0.06 & 0.02 \\
\hline$\tilde{\chi}_{2}^{0} \tilde{\chi}_{1}^{+}$ & 0.17 & 0.08 & 0.03 & $\tilde{q}_{i} \tilde{\chi}_{1}^{0}, \tilde{q}_{i}^{*} \tilde{\chi}_{1}^{0}$ & 0.08 & 0.03 & 0.01 \\
\hline$\tilde{\chi}_{3}^{0} \tilde{x}_{2}^{+}$ & 0.05 & 0.03 & 0.01 & $\tilde{q}_{i} \tilde{\chi}_{2}^{0}, \tilde{q}_{i}^{*} \tilde{\chi}_{2}^{0}$ & 0.08 & 0.03 & 0.01 \\
\hline$\tilde{\chi}_{4}^{0} \tilde{\chi}_{2}^{+}$ & 0.05 & 0.03 & 0.01 & $\tilde{v}_{i} \tilde{e}_{j}^{*}, \tilde{v}_{i}^{*} \tilde{e}_{j}$ & 0.09 & 0.04 & 0.01 \\
\hline$\tilde{g} \tilde{g}$ & 0.20 & 0.05 & 0.01 & $H b \bar{b}$ & 2.76 & 0.85 & \\
\hline$\tilde{g} \tilde{\chi}_{1}^{0}$ & 0.03 & 0.01 & & $A b \bar{b}$ & 2.73 & 0.84 & \\
\hline$\tilde{g} \tilde{\chi}_{2}^{0}$ & 0.03 & 0.01 & & $H^{+} b \bar{t}+h . c$. & 1.32 & 0.42 & \\
\hline$\tilde{g} \tilde{\chi}_{1}^{+}$ & 0.07 & 0.03 & 0.01 & $H^{+} W^{-}$ & 0.38 & 0.12 & \\
\hline$\tilde{q}_{i} \tilde{q}_{j}, \tilde{q}_{i} \tilde{q}_{j}^{*}$ & 3.70 & 1.51 & 0.53 & $H Z$ & 0.09 & 0.03 & \\
\hline$\tilde{\chi}_{1}^{+} \tilde{\chi}_{1}^{-}$ & 0.10 & 0.05 & 0.02 & $A Z$ & 0.09 & 0.03 & \\
\hline$\tilde{\chi}_{2}^{+} \tilde{\chi}_{2}^{-}$ & 0.03 & 0.02 & 0.01 & & & & \\
\hline$\tilde{e}_{i} \tilde{e}_{j}^{*}$ & 0.23 & 0.13 & 0.05 & & & & \\
\hline$\tilde{q}_{i} \tilde{g}, \tilde{q}_{i}^{*} \tilde{g}$ & 2.26 & 0.75 & 0.20 & & & & \\
\hline
\end{tabular}

$$
\begin{aligned}
& f \operatorname{Tr}\left(\lambda q^{c} q\right)+\frac{1}{6} f^{\prime} \epsilon_{i j k} \epsilon_{a b c} \\
& \quad \times\left(\lambda_{i a} \lambda_{j b} \lambda_{k c}+q_{i a}^{c} q_{j b}^{c} q_{k c}^{c}+q_{i a} q_{j b} q_{k c}\right),
\end{aligned}
$$

with $f$ and $f^{\prime}$ the corresponding Yukawa couplings. The superfields $\left(\tilde{N}, \tilde{N}^{c}\right)$ obtain vev's and provide masses to leptons and quarks

$m_{d}=f\langle\tilde{N}\rangle, \quad m_{u}=f\left\langle\tilde{N}^{c}\right\rangle, \quad m_{e}=f^{\prime}\langle\tilde{N}\rangle, \quad m_{v}=f^{\prime}\left\langle\tilde{N}^{c}\right\rangle$.

Having three families, $11 f$ couplings and $10 f^{\prime}$ couplings are present in the most general superpotential. Demanding the vanishing of all superfield anomalous dimensions, 9 conditions are imposed

$\sum_{j, k} f_{i j k}\left(f_{l j k}\right)^{*}+\frac{2}{3} \sum_{j, k} f_{i j k}^{\prime}\left(f_{l j k}^{\prime}\right)^{*}=\frac{16}{9} g^{2} \delta_{i l}$,

where

$f_{i j k}=f_{j k i}=f_{k i j}, \quad f_{i j k}^{\prime}=f_{j k i}^{\prime}=f_{k i j}^{\prime}=f_{i k j}^{\prime}=f_{k j i}^{\prime}=f_{j i k}^{\prime}$.

The masses of leptons and quarks are acquired from the vev's of the scalar parts of the superfields $\tilde{N}_{1,2,3}$ and $\tilde{N}_{1,2,3}^{c}$.

At $M_{\mathrm{GUT}}$ the $S U(3)^{3}$ FUT breaks ${ }^{5}$ to the MSSM, where as was already mentioned, both Higgs doublets couple mostly

\footnotetext{
$5[163,164]$ and Refs. therein discuss in detail the spontaneous breaking of $S U(3)^{3}$.
}

to the third generation. The FUT breaking leaves its mark in the form of Eq. (43), i.e. boundary conditions on the gauge and Yukawa couplings, the relation among the soft trilinear coupling, the corresponding Yukawa coupling and the unified gaugino mass and finally the soft scalar mass sum rule at $M_{\mathrm{GUT}}$. In this specific model the sum rule takes the form:

$m_{H_{u}}^{2}+m_{\tilde{t}^{c}}^{2}+m_{\tilde{q}}^{2}=M^{2}=m_{H_{d}}^{2}+m_{\tilde{b}^{c}}^{2}+m_{\tilde{q}^{2}}^{2}$.

The model is finite to all-orders if the solution of Eq. (43) is both isolated and unique. Then, $f^{\prime}=0$ and we have the relations

$f^{2}=f_{111}^{2}=f_{222}^{2}=f_{333}^{2}=\frac{16}{9} g^{2}$

Since all $f^{\prime}$ vanish, at one-loop order, the lepton masses vanish. Since these masses, even radiatively, cannot be produced because of the finiteness conditions, we are faced with a problem which needs further study. If the solution of Eq. (43) is unique but not isolated (i.e. parametric), we can have non zero $f^{\prime}$ leading to non-vanishing lepton masses and at the same time achieving two-loop finiteness. In that case the set of conditions restricting the Yukawa couplings read:

$f^{2}=r\left(\frac{16}{9}\right) g^{2}, \quad f^{\prime 2}=(1-r)\left(\frac{8}{3}\right) g^{2}$,

where $r$ parametrises the different solutions and as such is a free parameter. It should be noted that we use the sum rule as boundary condition for the soft scalar masses.

In our analysis we consider the two-loop finite version of the model, where again below $M_{\mathrm{GUT}}$ we get the MSSM. We 

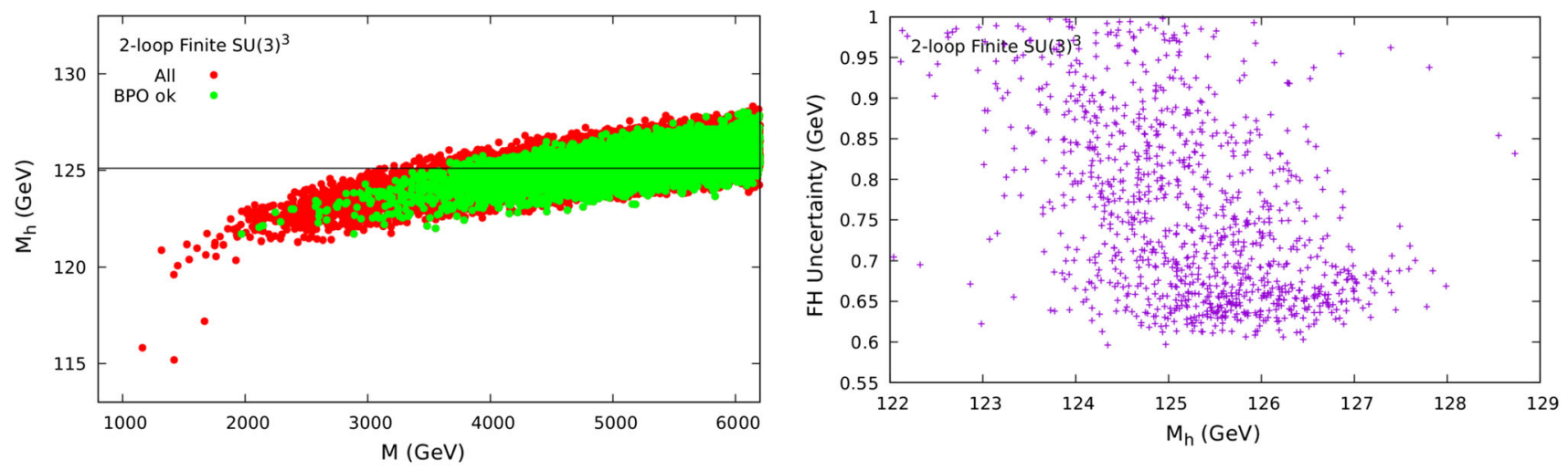

Fig. 4 Scatter plot for the finite $N=1 S U(3)^{3}$ model. Left: $M_{h}$ as a function of $M$. Right: the Higgs mass theoretical uncertainty [43]

take into account two new thresholds for the masses of the new particles at $\sim 10^{13} \mathrm{GeV}$ and $\sim 10^{14} \mathrm{GeV}$ resulting in a wider phenomenologically viable parameter space [156].

Looking for the values of the parameter $r$ which comply with the experimental limits, we find that both the top and bottom masses are in the experimental range (within $2 \sigma$ ) for the same value of $r$ between 0.65 and 0.80 (we singled out the $\mu<0$ case as the most promising). The inclusion of the above-mentioned thresholds gives an important improvement on the top mass from past versions of the model [158,165-167].

Figure 4 (left) shows the scatter plot of the light Higgs boson mass (green points satisfy the B-physics constraints), while the point-by-point calculated theoretical uncertainty is presented in Fig. 4 (right). The scattered points are due to the fact that we vary five parameters, namely $r$ and four of the parameters that form the sum rule. The uncertainty is found in the range between $0.6 \mathrm{GeV}$ and $1.0 \mathrm{GeV}$. All constraints regarding quark masses, the light Higgs boson mass and B-physics are satisfied, rendering the model very successful. The prediction of the SUSY spectrum results in relatively heavy particles, in full agreement with the current experimental searches.

Again, we choose three benchmarks, each featuring the LSP above $1500 \mathrm{GeV}, 2000 \mathrm{GeV}$ and $2400 \mathrm{GeV}$ respectively (but the LSP can go as high as $\sim 4100 \mathrm{GeV}$, again with too small cross sections). The input and output of SPheno 4.0.4 $[115,116]$ can be found in Tables 7 and 8 respectively (with the notation as in Sect. 5).

Like the previous models, the CDM relic density fails to comply with the experimental bounds (see Eq. (30)). The lightest neutralino is the LSP and considered as a CDM candidate, but its relic density does not go below 0.15 , since it is strongly Bino-like and would require a lower scale of the particle spectrum. It should be noted that if the B-physics constraints allowed for a unified gaugino mass $\sim 0.5 \mathrm{TeV}$ lower, then agreement with the CDM bounds as well could be achieved (see [44]). However, the alternatives proposed in the previous sections can be applied in this case as well, preserving finiteness.

It should be noted that in this model the scale of the heavy Higgs bosons does not vary monotonously with $M_{\tilde{\chi}_{1}^{0}}$, as in the previously considered models. This can be understood as follows. The Higgs bosons masses are determined by a combination of the sum rule at the unification scale, and the requirement of successful electroweak symmetry breaking at the low scale. Like in the finite scenario of the previous section, there are no direct relations between the soft scalar masses and the unified gaugino mass, but they are related through the corresponding sum rule and thus vary correlatedly, a fact that makes the dependence on the boundary values more restrictive. Furthermore (and even more importantly), the fact that we took into account the two thresholds at $\sim 10^{13} \mathrm{GeV}$ and $\sim 10^{14} \mathrm{GeV}$ (as mentioned above), allows the new particles, mainly the Higgsinos of the two other families (that were considered decoupled at the unification scale in previous analyses) and the down-like exotic quarks (in a lower degree), to affect the running of the (soft) RGEs in a non-negligible way. Thus, since at low energies the heavy Higgs masses depend mainly on the values of $m_{H_{u}}^{2}, m_{H_{d}}^{2},|\mu|$ and $\tan \beta$, they are substantially less connected to $M_{\tilde{\chi}_{1}^{0}}$ than in the other models, leading to a different exclusion potential, as will be discussed in the following.

Scenarios of finite $S U(3)^{3}$ are beyond the reach of the HL-LHC. Not only superpartners are too heavy, but also heavy Higgs bosons with a mass scale of $\sim 7 \mathrm{TeV}$ cannot be detected at the HL-LHC. At $100 \mathrm{TeV}$ collider (see Table 9), on the other hand, all three benchmark points are well within the reach of the $H / A \rightarrow \tau^{+} \tau^{-}$as well as the $H^{ \pm} \rightarrow \tau \nu_{\tau}, t b$ searches $[139,140]$, despite the slightly smaller values of $\tan \beta \sim 45$. This is particularly because of the different dependence of the heavy Higgs-boson mass scale on $M_{\tilde{\chi}_{1}^{0}}$, as discussed above. However, we have checked that $M_{A}$ can go up to to $\sim 11 \mathrm{TeV}$, and thus the heaviest part of the possible spectrum would escape the heavy Higgs-boson searches at the FCC-hh. 
Table 7 Finite $N=1 S U(3)^{3}$ predictions that are used as input to SPheno. Mass parameters are in $\mathrm{GeV}$ and rounded to $1 \mathrm{GeV}$

\begin{tabular}{lllllllllll}
\hline & $M_{1}$ & $M_{2}$ & $M_{3}$ & $|\mu|$ & $b$ & $A_{u}$ & $A_{d}$ & $A_{e}$ & $\tan \beta$ & $m_{Q_{1,2}}^{2}$ \\
\hline FSU33-1 & 1522 & 2758 & 6369 & 6138 & $1002^{2}$ & 4520 & 4413 & 1645 & 46.2 & $5574^{2}$ \\
FSU33-2 & 2070 & 3722 & 8330 & 7129 & $1083^{2}$ & 5841 & 5734 & 2357 & 45.5 & $7255^{2}$ \\
FSU33-3 & 2500 & 4484 & 10016 & 6790 & $972^{2}$ & 7205 & 7110 & 2674 & 49.7 & 8709 \\
\hline FSU33-1 & $m_{Q_{3}}^{2}$ & $m_{L_{1,2}}^{2}$ & $m_{L_{3}}^{2}$ & $m_{\bar{u}_{1,2}}^{2}$ & $m_{\bar{u}_{3}}^{2}$ & $m_{\bar{d}_{1,2}}^{2}$ & $m_{\bar{d}_{3}}^{2}$ & $m_{\bar{e}_{1,2}}^{2}$ & $m_{\bar{e}_{3}}^{2}$ \\
\hline FSU33-2 & $7705^{2}$ & $2382^{2}$ & $3754^{2}$ & $5234^{2}$ & $5548^{2}$ & $5197^{2}$ & $7043^{2}$ & $1558^{2}$ & $3095^{2}$ \\
FSU33-3 & $7255^{2}$ & $3136^{2}$ & $4131^{2}$ & $6749^{2}$ & $7225^{2}$ & $6745^{2}$ & $8523^{2}$ & $2238^{2}$ & $3342^{2}$ \\
\hline
\end{tabular}

Table 8 Masses for each benchmark of the finite $N=1 S U(3)^{3}$ (in TeV)

\begin{tabular}{|c|c|c|c|c|c|c|c|c|c|c|}
\hline & $M_{H}$ & $M_{A}$ & $M_{H^{ \pm}}$ & $M_{\tilde{g}}$ & $M_{\tilde{\chi}_{1}^{0}}$ & $M_{\tilde{\chi}_{2}^{0}}$ & $M_{\tilde{\chi}_{3}^{0}}$ & $M_{\tilde{\chi}_{4}^{0}}$ & $M_{\tilde{\chi}_{1}^{ \pm}}$ & $M_{\tilde{\chi}_{2}^{ \pm}}$ \\
\hline FSU33-1 & 7.029 & 7.029 & 7.028 & 6.526 & 1.506 & 2.840 & 6.108 & 6.109 & 2.839 & 6.109 \\
\hline FSU33-2 & 6.484 & 6.484 & 6.431 & 8.561 & 2.041 & 3.817 & 7.092 & 7.093 & 3.817 & 7.093 \\
\hline \multirow[t]{2}{*}{ FSU33-3 } & 6.539 & 6.539 & 6.590 & 10.159 & 2.473 & 4.598 & 6.780 & 6.781 & 4.598 & 6.781 \\
\hline & $M_{\tilde{e}_{1,2}}$ & $M_{\tilde{\nu}_{1,2}}$ & $M_{\tilde{\tau}}$ & $M_{\tilde{v}_{\tau}}$ & $M_{\tilde{d}_{1,2}}$ & $M_{\tilde{u}_{1,2}}$ & $M_{\tilde{b}_{1}}$ & $M_{\tilde{b}_{2}}$ & $M_{\tilde{t}_{1}}$ & $M_{\tilde{t}_{2}}$ \\
\hline FSU33-1 & 2.416 & 2.415 & 1.578 & 2.414 & 5.375 & 5.411 & 4.913 & 5.375 & 4.912 & 5.411 \\
\hline FSU33-2 & 3.188 & 3.187 & 2.269 & 3.186 & 7.026 & 7.029 & 6.006 & 7.026 & 6.005 & 7.029 \\
\hline FSU33-3 & 3.883 & 3.882 & 2.540 & 3.882 & 8.334 & 8.397 & 7.227 & 8.334 & 7.214 & 7.409 \\
\hline
\end{tabular}

Interesting are also the prospects for production of squark pairs and squark-gluino, which can reach $\sim 20 \mathrm{fb}$ for the FSU33-1 case, going down to a few fb for FSU33-2 and FSU33-3 scenarios. The lightest squarks decay almost exclusively to the third generation quark and chargino/neutralino, while gluino enjoys many possible decay channels to quarksquark pairs each one with branching fraction of the order of a percent, with the biggest one $\sim 20 \%$ to $t \tilde{t}_{1}+$ h.c..

We briefly discuss the SUSY discovery potential at the FCC-hh, referring agian to [141] with the same Figures as discussed in Sect. 5. Stops in FSU33-1 and FSU33-2 can be tested at the FCC-hh, while the masses turn out to be too heavy in FSU33-3. The situation is better for scalar quarks, where all three scenarios can be tested, but will not allow for a $5 \sigma$ discovery. Even more favorable are the prospects for gluino. Possibly all three scenarios can be tested at the $5 \sigma$ level. As in the previous scenario, the charginos and neutralinos will not be accessible, due to the too heavy LSP. Keeping in mind that only the lower part of possible mass spectrum is represented by the three benchmarks (with the LSP up to $\sim 1.5 \mathrm{TeV}$ heavier), we conclude that as before large parts of the parameter space will not be testable at the FCC-hh. The only partial exception here is the Higgs-boson sector, where only the the part with the highest possible Higgs-boson mass spectra would escape the FCC-hh searches.

\section{The reduced MSSM}

We finish our phenomenological analyses with the application of the method of coupling reduction to a version of the MSSM, where a covering GUT is assumed. The original partial reduction can be found in Refs. [168,169] where only the third fermionic generation is considered. Following this restriction, the superpotential reads:

$W=Y_{t} H_{2} Q t^{c}+Y_{b} H_{1} Q b^{c}+Y_{\tau} H_{1} L \tau^{c}+\mu H_{1} H_{2}$,

where $Y_{t, b, \tau}$ refer only to the third family, and the SSB Lagrangian is given by by (with the trilinear couplings $h_{t, b, \tau}$ for the third family)

$$
\begin{aligned}
-\mathcal{L}_{\mathrm{SSB}}= & \sum_{\phi} m_{\phi}^{2} \hat{\phi^{*}} \hat{\phi}+\left[m_{3}^{2} \hat{H}_{1} \hat{H}_{2}+\sum_{i=1}^{3} \frac{1}{2} M_{i} \lambda_{i} \lambda_{i}+\text { h.c }\right] \\
& +\left[h_{t} \hat{H_{2}} \hat{Q} \hat{t^{c}}+h_{b} \hat{H}_{1} \hat{Q} \hat{b}^{c}+h_{\tau} \hat{H}_{1} \hat{L} \hat{\tau}^{c}+\text { h.c. }\right]
\end{aligned}
$$

We start with the dimensionless sector and consider initially the top and bottom Yuakwa couplings and the strong gauge coupling. The rest of the couplings will be treated as corrections. If $Y_{(t, b)}^{2} /(4 \pi) \equiv \alpha_{(t, b)}$, the REs and 
Table 9 Expected production cross sections (in fb) for SUSY particles in the FSU33 scenarios

\begin{tabular}{llllllll}
\hline Scenarios $\sqrt{s}$ & $\begin{array}{l}\text { FSU33-1 } \\
100 \mathrm{TeV}\end{array}$ & $\begin{array}{l}\text { FSU33-2 } \\
100 \mathrm{TeV}\end{array}$ & $\begin{array}{l}\text { FSU33-3 } \\
100 \mathrm{TeV}\end{array}$ & Scenarios $\sqrt{s}$ & $\begin{array}{c}\text { FSU33-1 } \\
100 \mathrm{TeV}\end{array}$ & $\begin{array}{c}\text { FSU33-2 } \\
100 \mathrm{TeV}\end{array}$ & $\begin{array}{c}\text { FSU33-3 } \\
100 \mathrm{TeV}\end{array}$ \\
\hline$\tilde{\chi}_{1}^{0} \tilde{\chi}_{1}^{0}$ & 0.04 & 0.01 & 0.01 & $\tilde{q}_{i} \tilde{g}, \tilde{q}_{i}^{*} \tilde{g}$ & 22.12 & 3.71 & 1.05 \\
$\tilde{\chi}_{2}^{0} \tilde{\chi}_{2}^{0}$ & 0.04 & 0.01 & & $\tilde{v}_{i} \tilde{v}_{j}^{*}$ & 0.10 & 0.03 & 0.01 \\
$\tilde{\chi}_{2}^{0} \tilde{\chi}_{1}^{+}$ & 0.58 & 0.16 & 0.07 & $\tilde{u}_{i} \tilde{\chi}_{1}^{-}, \tilde{d}_{i} \tilde{\chi}_{1}^{+}+$h.c. & 1.22 & 0.25 & 0.08 \\
$\tilde{\chi}_{3}^{0} \tilde{\chi}_{2}^{+}$ & 0.02 & 0.01 & 0.01 & $\tilde{q}_{i} \tilde{\chi}_{1}^{0}, \tilde{q}_{i}^{*} \tilde{\chi}_{1}^{0}$ & 0.55 & 0.13 \\
$\tilde{\chi}_{4}^{0} \tilde{\chi}_{2}^{+}$ & 0.02 & 0.01 & 0.01 & $\tilde{q}_{i} \tilde{\chi}_{2}^{0}, \tilde{q}_{i}^{*} \tilde{\chi}_{2}^{0}$ & 0.60 & 0.13 & 0.05 \\
$\tilde{g} \tilde{g}$ & 2.61 & 0.30 & 0.07 & $\tilde{v}_{i} \tilde{e}_{j}^{*}, \tilde{v}_{i}^{*} \tilde{e}_{j}$ & 0.36 & 0.12 & 0.04 \\
$\tilde{g} \tilde{\chi}_{1}^{0}$ & 0.20 & 0.05 & 0.02 & $H b \bar{b}$ & 0.71 & 0.72 & 1.23 \\
$\tilde{g} \tilde{\chi}_{2}^{0}$ & 0.20 & 0.04 & 0.01 & $A b \bar{b}$ & 0.37 & 0.75 & 1.23 \\
$\tilde{g} \tilde{\chi}_{1}^{+}$ & 0.42 & 0.09 & 0.03 & $H^{+} b \bar{t}+h . c$. & 0.10 & 0.25 \\
$\tilde{q}_{i} \tilde{q}_{j}, \tilde{q}_{i} \tilde{q}_{j}^{*}$ & 25.09 & 6.09 & 2.25 & $H^{+} W^{-}$ & 0.02 & 0.04 \\
$\tilde{\chi}_{1}^{+} \tilde{\chi}_{1}^{-}$ & 0.37 & 0.10 & 0.04 & $H Z$ & 0.02 & 0.04 \\
$\tilde{e}_{i} \tilde{e}_{j}^{*}$ & 0.39 & 0.12 & 0.06 & $A Z$ & 0.19 \\
\hline
\end{tabular}

the Yukawa RGEs give

$\alpha_{i}=G_{i}^{2} \alpha_{3}, \quad$ where $G_{i}^{2}=\frac{1}{3}, \quad i=t, b$.

If the tau Yukawa is included in the reduction, the corresponding $G^{2}$ coefficient for tau turns negative [170], explaining why this coupling is treated also as a correction (i.e. it cannot be reduced).

We assume that the ratios of the top and bottom Yukawa to the strong coupling are constant at the GUT scale, i.e. they have negligible scale dependence,

$\frac{d}{d g_{3}}\left(\frac{Y_{t, b}^{2}}{g_{3}^{2}}\right)=0$.

Then, including the corrections from the $S U(2), U(1)$ and tau couplings, at the GUT scale, the coefficients $G_{t, b}^{2}$ become:

$G_{t}^{2}=\frac{1}{3}+\frac{71}{525} \rho_{1}+\frac{3}{7} \rho_{2}+\frac{1}{35} \rho_{\tau}$,

$G_{b}^{2}=\frac{1}{3}+\frac{29}{525} \rho_{1}+\frac{3}{7} \rho_{2}-\frac{6}{35} \rho_{\tau}$,

where

$\rho_{1,2}=\frac{g_{1,2}^{2}}{g_{3}^{2}}=\frac{\alpha_{1,2}}{\alpha_{3}}, \quad \rho_{\tau}=\frac{g_{\tau}^{2}}{g_{3}^{2}}=\frac{\frac{Y_{\tau}^{2}}{4 \pi}}{\alpha_{3}}$.

We shall treat Eq. (50) as boundary conditions at the GUT scale.

Going to the two-loop level, we assume that the corrections take the following form:

$\alpha_{i}=G_{i}^{2} \alpha_{3}+J_{i}^{2} \alpha_{3}^{2}, \quad i=t, b$.
Then, the two-loop coefficients, $J_{i}$, including the corrections from the gauge and the tau Yukawa couplings, are:

$J_{t}^{2}=\frac{1}{4 \pi} \frac{N_{t}}{D}, \quad J_{b}^{2}=\frac{1}{4 \pi} \frac{N_{b}}{5 D}$,

where $D, N_{t}$ and $N_{b}$ are known quantities which can be found in Ref. [171].

Proceeding to the the SSB Lagrangian, Eq. (49), and the dimension-one parameters, i.e the trilinear couplings $h_{t, b, \tau}$, we first reduce $h_{t, b}$ and we get

$h_{i}=c_{i} Y_{i} M_{3}=c_{i} G_{i} M_{3} g_{3}, \quad$ where $c_{i}=-1 \quad i=t, b$,

where $M_{3}$ is the gluino mass. Adding the corrections from the gauge and the tau couplings we have

$c_{t}=-\frac{A_{A} A_{b b}+A_{t b} B_{B}}{A_{b t} A_{t b}-A_{b b} A_{t t}}, \quad c_{b}=-\frac{A_{A} A_{b t}+A_{t t} B_{B}}{A_{b t} A_{t b}-A_{b b} A_{t t}}$.

Again, $A_{t t}, A_{b b}$ and $A_{t b}$ can be found in Ref. [171].

We end up with the soft scalar masses $m_{\phi}^{2}$ of the SSB Lagrangian. Assuming the relations $m_{i}^{2}=c_{i} M_{3}^{2}(i=$ $Q, u, d, H_{u}, H_{d}$ ), and adding the corrections from the gauge, the tau couplings and $h_{\tau}$, we get

$c_{Q}=-\frac{c_{Q \mathrm{Num}}}{D_{m}}, \quad c_{u}=-\frac{1}{3} \frac{c_{u \mathrm{Num}}}{D_{m}}, \quad c_{d}=-\frac{c_{d \mathrm{Num}}}{D_{m}}$,

$c_{H_{u}}=-\frac{2}{3} \frac{c_{H_{u} \mathrm{Num}}}{D_{m}}, \quad c_{H_{d}}=-\frac{c_{H_{d} \mathrm{Num}}}{D_{m}}$,

where $D_{m}, c_{Q \mathrm{Num}}, c_{u \mathrm{Num}}, c_{d \mathrm{Num}}, c_{H_{u} \mathrm{Num}}, c_{H_{d} \mathrm{Num}}$ and the complete analysis are again given in Ref. [171]. These values do not obey any soft scalar mass sum rule.

If only the reduced system was used, i.e. the strong, top and bottom Yukawa couplings as well as the $h_{t}$ and $h_{b}$, the 



Fig. 5 Left: the lightest Higgs boson mass, $M_{h}$ in the Reduced MSSM. The green points is the full model prediction. Right: the lightest Higgs mass theoretical uncertainty [43]

coefficients turn to be

$c_{Q}=c_{u}=c_{d}=\frac{2}{3}, \quad c_{H_{u}}=c_{H_{d}}=-1 / 3$,

which clearly obey the sum rules

$\frac{m_{Q}^{2}+m_{u}^{2}+m_{H_{u}}^{2}}{M_{3}^{2}}=c_{Q}+c_{u}+c_{H_{u}}=1$,

$\frac{m_{Q}^{2}+m_{d}^{2}+m_{H_{d}}^{2}}{M_{3}^{2}}=c_{Q}+c_{d}+c_{H_{d}}=1$.

There is an essential point for the gaugino masses that should be mentioned. The application of the Hisano-Shifman relation, Eq. (12), is made for each gaugino mass as a boundary condition with unified gauge coupling at $M_{\mathrm{GUT}}$. Then, at one-loop level, the gaugino mass depends on the one-loop coefficient of the corresponding $\beta$-function and an arbitrary mass $M_{0}, \quad M_{i}=b_{i} M_{0}$. This fact permits, with a suitable choice of $M_{0}$, to have the gluino mass equal to the unified gaugino mass, while the gauginos masses of the other two gauge groups are given by the gluino mass multiplied by the ratio of the appropriate one-loop $\beta$ coefficient.

For our analysis we choose the unification scale to apply the corrections to all these RGI relations. The full discussion on the selection of the free parameters of the model can be found in [44]. In total, we vary $\rho_{\tau}, \rho_{h_{\tau}}, M$ and $\mu$. This results in the scattered points of the next figure.

The model's predictions for the bottom and top mass lie within $2 \sigma$ of Eq. (25). The scatter plot of the light Higgs boson mass $M_{h}$ is shown in Fig. 5 (left), while the theory uncertainty given in Fig. 5 (right) has dropped below $1 \mathrm{GeV}$. The Higgs mass predicted by the model lies perfectly in the experimentally measured range.

The $M_{h}$ limits set a limit on the low-energy supersymmetric masses, which we briefly discuss. The three selected benchmarks correspond to $\overline{\mathrm{DR}}$ pseudoscalar Higgs boson masses above $1900 \mathrm{GeV}, 1950 \mathrm{GeV}$ and $2000 \mathrm{GeV}$ respec- tively. The input of SPheno 4.0.4 [115,116] can be found in Table 10 (notation as in Sect. 5).

Table 11 shows the resulting masses of Higgs bosons and some of the lightest SUSY particles. The lightest neutralino (LSP) is Wino-like, as imposed by the Hisano-Shifman relation, Eq. (12), and thus the CDM relic density is below the boundaries of Eq. (30). This renders this model viable if Eq. (30) is applied only as an upper limit and additional sources of CDM are allowed. An additional DM component could be, e.g., a SUSY axion [172], which would then bring the total DM density into agreement with the Planck measurement of $\Omega_{\mathrm{CDM}} h^{2}$. This is in contrast to the other three models discussed above.

In addition, there is one more point that should be stressed. We find $M_{A} \lesssim 1.5 \mathrm{TeV}$ (for large values of $\tan \beta$ as in the other models), values substantially lower than in the previously considered models. This can be understood as follows. In this model, we have direct relations between the soft scalar masses and the unified gaugino mass, which receive corrections from the two gauge couplings $g_{1}$ and $g_{2}$ and the Yukawa coupling of the $\tau$ lepton. As mentioned above, in the absence of these corrections the relations obey the soft scalar mass sum rule. However, unlike all the previous models, these corrections make the sum rule only approximate. Thus, these unique boundary conditions result in very low values for the masses of the heavy Higgs bosons (even compared to the minimal $S U(5)$ case presented above, which also exhibits direct relations which however obey the sum rule). A relatively light spectrum is also favored by the prediction for the light CP-even Higgs boson mass, which turns out to be relatively high in this model and does not allow us to consider heavier spectra. Thus, in this model, contrary to the models analyzed before, because of the large $\tan \beta \sim 45$ found here, the physical mass of the pseudoscalar Higgs boson, $M_{A}$, is excluded by the searches $H / A \rightarrow \tau \tau$ at ATLAS with 139/fb [91] for all three benchmarks. One could try considering a heavier spectrum, in which we would have $M_{A} \gtrsim 1900 \mathrm{GeV}$, 
Table 10 Reduced MSSM predictions that are used as input to SPheno mass parameters are in $\mathrm{GeV}$ and rounded to $1 \mathrm{GeV}$

\begin{tabular}{lllllllllll}
\hline & $M_{1}$ & $M_{2}$ & $M_{3}$ & $|\mu|$ & $b$ & $A_{u}$ & $A_{d}$ & $A_{e}$ & $\tan \beta$ & $m_{Q_{1,2}}^{2}$ \\
\hline RMSSM-1 & 3711 & 1014 & 7109 & 4897 & $284^{2}$ & 5274 & 5750 & 20 & 44.9 & $5985^{2}$ \\
RMSSM-2 & 3792 & 1035 & 7249 & 4983 & $294^{2}$ & 5381 & 5871 & 557 & 44.6 & $6103^{2}$ \\
RMSSM-3 & 3829 & 1045 & 7313 & 5012 & $298^{2}$ & 5427 & 5942 & 420 & 45.3 & $6161^{2}$ \\
\hline & $m_{Q_{3}}^{2}$ & $m_{L_{1,2}}^{2}$ & $m_{L_{3}}^{2}$ & $m_{\bar{u}_{1,2}}^{2}$ & $m_{\bar{u}_{3}}^{2}$ & $m_{\bar{d}_{1,2}}^{2}$ & $m_{\bar{d}_{3}}^{2}$ & $m_{\bar{e}_{1,2}}^{2}$ & $m_{\bar{e}_{3}}^{2}$ \\
\hline RMSSM-1 & $5545^{2}$ & $2106^{2}$ & $2069^{2}$ & $6277^{2}$ & $5386^{2}$ & $5989^{2}$ & $5114^{2}$ & $3051^{2}$ & $4491^{2}$ \\
RMSSM-2 & $5656^{2}$ & $2122^{2}$ & $2290^{2}$ & $6385^{2}$ & $5476^{2}$ & $6110^{2}$ & $5219^{2}$ & $3153^{2}$ & $4181^{2}$ \\
RMSSM-3 & $5708^{2}$ & $2106^{2}$ & $2279^{2}$ & $6427^{2}$ & $5506^{2}$ & $6172^{2}$ & $5269^{2}$ & $3229^{2}$ & $3504^{2}$ \\
\hline
\end{tabular}

Table 11 Masses for each benchmark of the reduced MSSM (in TeV)

\begin{tabular}{|c|c|c|c|c|c|c|c|c|c|c|}
\hline & $M_{H}$ & $M_{A}$ & $M_{H^{ \pm}}$ & $M_{\tilde{g}}$ & $M_{\tilde{\chi}_{1}^{0}}$ & $M_{\tilde{\chi}_{2}^{0}}$ & $M_{\tilde{\chi}_{3}^{0}}$ & $M_{\tilde{\chi}_{4}^{0}}$ & $M_{\tilde{\chi}_{1}^{ \pm}}$ & $M_{\tilde{\chi}_{2}^{ \pm}}$ \\
\hline RMSSM-1 & 1.393 & 1.393 & 1.387 & 7.253 & 1.075 & 3.662 & 4.889 & 4.891 & 1.075 & 4.890 \\
\hline RMSSM-2 & 1.417 & 1.417 & 1.414 & 7.394 & 1.098 & 3.741 & 4.975 & 4.976 & 1.098 & 4.976 \\
\hline \multirow[t]{2}{*}{ RMSSM-3 } & 1.491 & 1.491 & 1.492 & 7.459 & 1.109 & 3.776 & 5.003 & 5.004 & 1.108 & 5.004 \\
\hline & $M_{\tilde{e}_{1,2}}$ & $M_{\tilde{\nu}_{1,2}}$ & $M_{\tilde{\tau}}$ & $M_{\tilde{v}_{\tau}}$ & $M_{\tilde{d}_{1,2}}$ & $M_{\tilde{u}_{1,2}}$ & $M_{\tilde{b}_{1}}$ & $M_{\tilde{b}_{2}}$ & $M_{\tilde{t}_{1}}$ & $M_{\tilde{t}_{2}}$ \\
\hline RMSSM-1 & 2.124 & 2.123 & 2.078 & 2.079 & 6.189 & 6.202 & 5.307 & 5.715 & 5.509 & 5.731 \\
\hline RMSSM-2 & 2.297 & 2.139 & 2.140 & 2.139 & 6.314 & 6.324 & 5.414 & 5.828 & 5.602 & 5.842 \\
\hline RMSSM-3 & 2.280 & 2.123 & 2.125 & 2.123 & 6.376 & 6.382 & 5.465 & 5.881 & 5.635 & 5.894 \\
\hline
\end{tabular}

but in that case the light Higgs mass would be well above its acceptable region. Particularly, it would be above 128 $\mathrm{GeV}$, a value that is clearly excluded, especially given the improved (much smaller) uncertainty calculated by the new FeynHiggs code). Thus, the current version of this model has been ruled out experimentally. Consequently, we do not show any SUSY or Higgs production cross sections.

\section{Conclusions}

The reduction of couplings scheme consists in searching for RGE relations among parameters of a renormalizable theory that hold to all orders in perturbation theory. In certain $N=1$ theories such a reduction of couplings indeed appears to be theoretically realised and therefore it developed to a powerful tool able to reduce the parameters and increase the predictivity of these theories. In the present paper first we briefly reviewed the ideas concerning the reduction of couplings of renormalizable theories and the theoretical methods which have been developed to confront the problem. Then we turned to the question of testing experimentally the idea of reduction of couplings. Four specific models, namely the reduced minimal $N=1 S U(5)$, the all-loop finite $N=1 S U(5)$, the two-loop Finite $N=1 S U(3)^{3}$ and the Reduced MSSM, have been considered for which new results have been obtained using the updated Higgsboson mass calculation of FeynHiggs. In each case benchmark points in the low-mass regions have been chosen for which the SPheno code has been used to calculate the spectrum of SUSY particles and their decay modes. Finally the MadGraph event generator was used to compute the production cross sections of relevant final states at the $14 \mathrm{TeV}$ (HL-)LHC and 100 TeV FCC-hh colliders.

The first three (unified) models were found to be in comfortable agreement with LHC measurements and searches, with the exception of the bottom quark mass in the Reduced Minimal $S U(5)$, for which agreement with measurements can be achieved only at the $4 \sigma$ level. In addition it was found that all models predict relatively heavy spectra, which evade largely the detection in the HL-LHC. We found one noticeable exception. The reduced MSSM features a relatively light heavy Higgs-boson mass spectrum. Together with the relatively high value of $\tan \beta$ this spectrum is excluded already by current searches at ATLAS and CMS for in the $p p \rightarrow H / A \rightarrow \tau^{+} \tau^{-}$mode. We also analyzed the accessibility of the SUSY and heavy Higgs spectrum at the FCC-hh with $\sqrt{s}=100 \mathrm{TeV}$. We found that the lower parts of the parameter space will be testable at the $2 \sigma$ level, with only an even smaller part discoverable at the $5 \sigma$ level. However, the heavier parts of the possible SUSY spectra will remain elusive even at the FCC-hh. One exception here is the heavy 
Higgs-boson sector of the two-loop finite $N=1 S U(3)^{3}$ model, which exhibits a spectrum where only the highest possible mass values could escape the searches at the FCC-hh.

Acknowledgements GZ thanks the ITP of Heidelberg, MPI Munich, CERN Department of Theoretical Physics, IFT Madrid and MPI-AEI for their hospitality. The work of SH is supported in part by the MEINCOP Spain under Contract FPA2016-78022-P and and under Contract PID2019-110058GB-C21, in part by the Spanish Agencia Estatal de Investigación (AEI), the EU Fondo Europeo de Desarrollo Regional (FEDER) through the project FPA2016-78645-P, in part by the "Spanish Red Consolider MultiDark" FPA2017-90566-REDC, and in part by the AEI through the Grant IFT Centro de Excelencia Severo Ochoa SEV2016-0597. The work of MM is partly supported by UNAM PAPIIT through Grant IN111518. The work of GP, NT and GZ is partially supported by the COST action CA16201, GZ is also partially supported by the Grant DEC-2018/31/B/ST2/02283 of NSC, Poland. GZ has been supported within the Excellence Initiative funded by the German and State Governments, at the Institute for Theoretical Physics, Heidelberg University and from the Excellence Grant Enigmass of LAPTh. The work of JK and WK has been supported $b$ the National Science Centre, Poland, the HARMONIA project under contract UMO2015/18/M/ST2/00518 (2016-2020).

Data Availability Statement This manuscript has no associated data or the data will not be deposited. [Authors' comment: All relative data and results are displayed in the appropriate tables, there is no extra data to deposit.]

Open Access This article is licensed under a Creative Commons Attribution 4.0 International License, which permits use, sharing, adaptation, distribution and reproduction in any medium or format, as long as you give appropriate credit to the original author(s) and the source, provide a link to the Creative Commons licence, and indicate if changes were made. The images or other third party material in this article are included in the article's Creative Commons licence, unless indicated otherwise in a credit line to the material. If material is not included in the article's Creative Commons licence and your intended use is not permitted by statutory regulation or exceeds the permitted use, you will need to obtain permission directly from the copyright holder. To view a copy of this licence, visit http://creativecomm ons.org/licenses/by/4.0/.

Funded by SCOAP ${ }^{3}$.

\section{References}

1. J. Kubo, S. Heinemeyer, M. Mondragón, O. Piguet, K. Sibold, W. Zimmermann, G. Zoupanos, PoS (Higgs \& top)001, Ed. Klaus Sibold, https://pos.sissa.it/cgi-bin/reader/conf.cgi? confid=222. A short version is published in arXiv: 1411.7155 [hep$\mathrm{ph}]$

2. W. Zimmermann, Commun. Math. Phys. 97, 211 (1985)

3. R. Oehme, W. Zimmermann, Commun. Math. Phys. 97, 569 (1985)

4. R. Oehme, Prog. Theor. Phys. Suppl. 86, 215 (1986)

5. E. Ma, Phys. Rev. D 17, 623 (1978)

6. E. Ma, Phys. Rev. D 31, 1143 (1985)

7. N.P. Chang, Phys. Rev. D 10, 2706 (1974)

8. S. Nandi, W.C. Ng, Phys. Rev. D 20, 972 (1979)

9. J.C. Pati, A. Salam, Phys. Rev. Lett. 31, 661 (1973)

10. H. Georgi, S.L. Glashow, Phys. Rev. Lett. 32, 438 (1974)

11. H. Georgi, H.R. Quinn, S. Weinberg, Phys. Rev. Lett. 33, 451 (1974)

12. H. Fritzsch, P. Minkowski, Ann. Phys. 93, 193 (1975)
13. F. Gursey, P. Ramond, P. Sikivie, Phys. Lett. 60B, 177 (1976)

14. Y. Achiman, B. Stech, Phys. Lett. 77B, 389 (1978)

15. D. Kapetanakis, M. Mondragón, G. Zoupanos, Z. Phys. C 60, 181 (1993)

16. M. Mondragón, G. Zoupanos, Nucl. Phys. Proc. Suppl. 37C, 98 (1995)

17. J. Kubo, M. Mondragón, G. Zoupanos, Nucl. Phys. B 424, 291 (1994)

18. J. Kubo, M. Mondragón, N.D. Tracas, G. Zoupanos, Phys. Lett. B 342, 155 (1995)

19. J. Kubo, M. Mondragón, G. Zoupanos, Published In: ICHEP 1994:0589-592, in Glasgow 1994, Proceedings, High energy physics, vol. 2, pp. 589-591. arXiv:hep-th/9409032

20. J. Kubo, M. Mondragón, G. Zoupanos, Published In: Trieste HEP Cosmology 1995:653-672, in Trieste 1995, High energy physics and cosmology, pp. 653-672, Contribution to: ICTP Summer School in High-energy Physics and Cosmology, 653-672. arXiv:hep-ph/9512400

21. J. Kubo, M. Mondragón, M. Olechowski, G. Zoupanos, Nucl. Phys. B 479, 25-45 (1996). arXiv:hep-ph/9512435

22. J. Kubo, M. Mondragón, M. Olechowski, G. Zoupanos, Published In: Brussels EPS HEP 1995:488-489, Contribution to: International Europhysics Conference on High-energy Physics (HEP 95), 488-489. arXiv:hep-ph/9510279

23. J. Kubo, M. Mondragón, S. Shoda, G. Zoupanos, Nucl. Phys. B 469, 3-20 (1996). arXiv:hep-ph/9512258

24. J. Kubo, M. Mondragón, G. Zoupanos, Published in: ICHEP 96, 1391-1394. arXiv:hep-ph/9702391

25. J. Kubo, M. Mondragón, G. Zoupanos, Acta Phys. Polon. B 27, 3911-3944 (1997)

26. T. Kobayashi, J. Kubo, M. Mondragón, G. Zoupanos, Nucl. Phys. B 511, 45 (1998)

27. J. Kubo, M. Mondragón, G. Zoupanos, arXiv:hep-ph/9708225

28. M. Mondragón, G. Zoupanos, J. Phys. Conf. Ser. 171, 012095 (2009)

29. Tevatron Electroweak Working Group, CDF and D0 Collaborations, (2011). arXiv: 1107.5255

30. S. Heinemeyer, M. Mondragón, G. Zoupanos, JHEP 0807, 135 (2008). arXiv:0712.3630 [hep-ph]

31. ATLAS Collaboration, G. Aad et al., Phys. Lett. B 716, 1 (2012). arXiv: 1207.7214

32. C.M.S. Collaboration, S. Chatrchyan et al., Phys. Lett. B 716, 30 (2012). arXiv:1207.7235

33. ATLAS Collaboration, Reports ATLAS-CONF-2013-014, ATLAS-COM-CONF-2013-025 (2013); CMS Collaboration, S. Chatrchyan et al., (2013). arXiv:1303.4571

34. G. Degrassi, S. Heinemeyer, W. Hollik, P. Slavich, G. Weiglein, Eur. Phys. J. C 28, 133 (2003). arXiv:0212020 [hep-ph]

35. H. Bahl, S. Heinemeyer, W. Hollik, G. Weiglein, Eur. Phys. J. C 78(1), 57 (2018). arXiv:1706.00346 [hep-ph]

36. S. Heinemeyer, W. Hollik, G. Weiglein, Comput. Phys. Commun. 124, 76 (2000). arXiv:hep-ph/9812320

37. S. Heinemeyer, W. Hollik, G. Weiglein, Eur. Phys. J. C 9, 343 (1999). arXiv:hep-ph/9812472

38. M. Frank, T. Hahn, S. Heinemeyer, W. Hollik, H. Rzehak, G. Weiglein, JHEP 0702, 047 (2007). arXiv:0611326 [hep-ph]

39. T. Hahn, S. Heinemeyer, W. Hollik, H. Rzehak, G. Weiglein, Comput. Phys. Commun. 180, 1426 (2009)

40. T. Hahn, S. Heinemeyer, W. Hollik, H. Rzehak, G. Weiglein, Phys. Rev. Lett. 112(14), 141801 (2014). arXiv:1312.4937 [hep-ph]

41. H. Bahl, W. Hollik, Eur. Phys. J. C 76(9), 499 (2016). arXiv:1608.01880 [hep-ph]

42. H. Bahl, T. Hahn, S. Heinemeyer, W. Hollik, S. Paßehr, H. Rzehak, G. Weiglein, arXiv:1811.09073 [hep-ph]; See http://www. feynhiggs.de 
43. H. Bahl, S. Heinemeyer, W. Hollik, G. Weiglein, Eur. Phys. J. C 80(6), 497 (2020). arXiv:1912.04199 [hep-ph]

44. S. Heinemeyer, M. Mondragón, G. Patellis, N. Tracas, G. Zoupanos, arXiv:2002.10983 [hep-ph]

45. J. Kubo, K. Sibold, W. Zimmermann, Nucl. Phys. B 259, 331 (1985)

46. J. Kubo, K. Sibold, W. Zimmermann, Phys. Lett. B 220, 185 (1989)

47. O. Piguet, K. Sibold, Phys. Lett. B 229, 83 (1989)

48. J. Kubo, M. Mondragón, G. Zoupanos, Phys. Lett. B 389, 523 (1996). arXiv:hep-ph/9609218

49. P. Breitenlohner, D. Maison, Commun. Math. Phys. 219, 179 (2001)

50. W. Zimmermann, Commun. Math. Phys. 219, 221 (2001)

51. A. Parkes, P.C. West, Phys. Lett. 138B, 99 (1984)

52. P.C. West, Phys. Lett. 137B, 371 (1984)

53. D.R.T. Jones, A.J. Parkes, Phys. Lett. 160B, 267 (1985)

54. D.R.T. Jones, L. Mezincescu, Phys. Lett. 138B, 293 (1984)

55. A.J. Parkes, Phys. Lett. 156B, 73 (1985)

56. J. Wess, B. Zumino, Phys. Lett. 49B, 52 (1974)

57. J. Iliopoulos, B. Zumino, Nucl. Phys. B 76, 310 (1974)

58. K. Fujikawa, W. Lang, Nucl. Phys. B 88, 61 (1975)

59. R. Delbourgo, Nuovo Cim. A 25, 646 (1975)

60. A. Salam, J.A. Strathdee, Nucl. Phys. B 86, 142 (1975)

61. M.T. Grisaru, W. Siegel, M. Rocek, Nucl. Phys. B 159, 429 (1979)

62. L. Girardello, M.T. Grisaru, Nucl. Phys. B 194, 65 (1982)

63. Y. Yamada, Phys. Rev. D 50, 3537 (1994). arXiv:hep-ph/9401241

64. D.I. Kazakov, Phys. Lett. B 421, 211 (1998). arXiv:hep-ph/9709465

65. I. Jack, D.R.T. Jones, A. Pickering, Phys. Lett. B 426, 73 (1998). arXiv:hep-ph/9712542

66. J. Hisano, M.A. Shifman, Phys. Rev. D 56, 5475 (1997). arXiv:hep-ph/9705417

67. I. Jack, D.R.T. Jones, Phys. Lett. B 415, 383 (1997). arXiv:hep-ph/9709364

68. L.V. Avdeev, D.I. Kazakov, I.N. Kondrashuk, Nucl. Phys. B 510, 289 (1998). arXiv:hep-ph/9709397

69. D.I. Kazakov, Phys. Lett. B 449, 201 (1999). arXiv:hep-ph/9812513

70. I. Jack, D.R.T. Jones, Phys. Lett. B 465, 148 (1999). arXiv:hep-ph/9907255

71. T. Kobayashi et al., AIP Conf. Proc. 490, 279 (1999)

72. T. Kobayashi, J. Kubo, G. Zoupanos, Phys. Lett. B 427, 291 (1998)

73. S. Rajpoot, J.G. Taylor, Phys. Lett. B 147, 91 (1984)

74. S. Rajpoot, J.G. Taylor, Int. J. Theor. Phys. 25, 117 (1986)

75. D.R.T. Jones, L. Mezincescu, Y.P. Yao, Phys. Lett. B 148, 317 (1984)

76. I. Jack, D.R.T. Jones, Phys. Lett. B 333, 372 (1994)

77. L. O'Raifeartaigh, Nucl. Phys. B 96, 331 (1975)

78. P. Fayet, J. Iliopoulos, Phys. Lett. B 51, 461 (1974)

79. C. Lucchesi, O. Piguet, K. Sibold, Phys. Lett. B 201, 241 (1988)

80. C. Lucchesi, O. Piguet, K. Sibold, Helv. Phys. Acta 61, 321 (1988)

81. O. Piguet, K. Sibold, Int. J. Mod. Phys. A 1, 913 (1986)

82. O. Piguet, K. Sibold, Phys. Lett. B 177, 373 (1986)

83. P. Ensign, K.T. Mahanthappa, Phys. Rev. D 36, 3148 (1987)

84. C. Lucchesi, G. Zoupanos, Fortschr. Phys. 45, 129 (1997)

85. O. Piguet, talk given at 10th International Conference on Problems of Quantum Field Theory. arXiv:hep-th/9606045

86. M. Tanabashi et al., [Particle Data Group], Phys. Rev. D 98(3), 030001 (2018)

87. S. Heinemeyer, O. Stal, G. Weiglein, Phys. Lett. B 710, 201 (2012). arXiv:1112.3026 [hep-ph]

88. P. Bechtle, S. Heinemeyer, O. Stal, T. Stefaniak, G. Weiglein, L. Zeune, Eur. Phys. J. C 73(4), 2354 (2013), arXiv:1211.1955 [hep-ph]
89. P. Bechtle, H.E. Haber, S. Heinemeyer, O. Stal, T. Stefaniak, G. Weiglein, L. Zeune, Eur. Phys. J. C 77(2), 67 (2017). arXiv:1608.00638 [hep-ph]

90. E. Bagnaschi, H. Bahl, J. Ellis, J. Evans, T. Hahn, S. Heinemeyer, W. Hollik, K.A. Olive, S. Paßehr, H. Rzehak et al., Eur. Phys. J. C 79(2), 149 (2019). arXiv:1810.10905 [hep-ph]

91. G. Aad et al., [ATLAS], Phys. Rev. Lett. 125(5), 051801 (2020). arXiv:2002.12223 [hep-ex]

92. M. Misiak et al., Phys. Rev. Lett. 98, 022002 (2007). arXiv:hep-ph/0609232

93. M. Ciuchini, G. Degrassi, P. Gambino, G.F. Giudice, Nucl. Phys. B 534, 3 (1998). arXiv:hep-ph/9806308

94. G. Degrassi, P. Gambino, G.F. Giudice, JHEP 0012, 009 (2000). arXiv:hep-ph/0009337

95. M. Carena, D. Garcia, U. Nierste, C.E.M. Wagner, Phys. Lett. B 499, 141 (2001). arXiv:hep-ph/0010003

96. G. D'Ambrosio, G.F. Giudice, G. Isidori, A. Strumia, Nucl. Phys. B 645, 155 (2002). arXiv:hep-ph/0207036

97. D. Asner et al., [Heavy Flavor Averaging Group], arXiv: 1010.1589 [hep-ex]

98. C. Bobeth, M. Gorbahn, T. Hermann, M. Misiak, E. Stamou, M. Steinhauser, Phys. Rev. Lett. 112, 101801 (2014). arXiv:1311.0903 [hep-ph]

99. T. Hermann, M. Misiak, M. Steinhauser, JHEP 1312, 097 (2013). arXiv:1311.1347 [hep-ph]

100. C. Bobeth, M. Gorbahn, E. Stamou, Phys. Rev. D 89(3), 034023 (2014). arXiv:1311.1348 [hep-ph]

101. A.J. Buras, Phys. Lett. B 566, 115 (2003). arXiv:hep-ph/0303060

102. G. Isidori, D.M. Straub, Eur. Phys. J. C 72, 2103 (2012). arXiv:1202.0464 [hep-ph]

103. R. Aaij et al., [LHCb Collaboration], Phys. Rev. Lett. 110, 021801 (2013). arXiv:1211.2674 [hep-ex]

104. S. Chatrchyan et al., [CMS Collaboration], Phys. Rev. Lett. 111, 101804 (2013). arXiv:1307.5025 [hep-ex]

105. CMS and LHCb Collaborations [CMS and LHCb Collaborations], CMS-PAS-BPH-13-007, LHCb-CONF-2013-012, CERNLHCb-CONF-2013-012

106. G. Isidori, P. Paradisi, Phys. Lett. B 639, 499 (2006). arXiv:hep-ph/0605012

107. G. Isidori, F. Mescia, P. Paradisi, D. Temes, Phys. Rev. D 75, 115019 (2007). arXiv:hep-ph/0703035

108. K.A. Olive et al., [Particle Data Group], Chin. Phys. C 38, 090001 (2014)

109. A.J. Buras, P. Gambino, M. Gorbahn, S. Jager, L. Silvestrini, Nucl. Phys. B 592, 55 (2001). arXiv:hep-ph/0007313

110. R. Aaij et al., [LHCb Collaboration], New J. Phys. 15, 053021 (2013). arXiv:1304.4741 [hep-ex]

111. H. Goldberg, Phys. Rev. Lett. 50, 1419 (1983) [Erratum: Phys. Rev. Lett. 103, 099905 (2009)]

112. J.R. Ellis, J.S. Hagelin, D.V. Nanopoulos, K.A. Olive, M. Srednicki, Nucl. Phys. B 238, 453 (1984). https://doi.org/10.1016/ 0550-3213(84)90461-9

113. N. Aghanim et al., Planck. Astron. Astrophys. 641, A6 (2020). https://doi.org/10.1051/0004-6361/201833910. arXiv:1807.06209 [astro-ph.CO]

114. F. Staub, Comput. Phys. Commun. 185, 1773-1790 (2014). arXiv: 1309.7223 [hep-ph]

115. W. Porod, Comput. Phys. Commun. 153, 275-315 (2003). arXiv:hep-ph/0301101

116. W. Porod, F. Staub, Comput. Phys. Commun. 183, 2458-2469 (2012). arXiv:1104.1573 [hep-ph]

117. M. Gabelmann, M. Mühlleitner, F. Staub, Eur. Phys. J. C 79(2), 163 (2019). arXiv:1810.12326 [hep-ph]

118. M. Goodsell, K. Nickel, F. Staub, Eur. Phys. J. C 75(6), 290 (2015). arXiv:1503.03098 [hep-ph] 
119. C. Degrande, C. Duhr, B. Fuks, D. Grellscheid, O. Mattelaer, T. Reiter, Comput. Phys. Commun. 183, 1201-1214 (2012). arXiv:1108.2040 [hep-ph]

120. F. Staub, Comput. Phys. Commun. 184, 1792-1809 (2013). arXiv:1207.0906 [hep-ph]

121. J. Alwall, R. Frederix, S. Frixione, V. Hirschi, F. Maltoni, O. Mattelaer, H.S. Shao, T. Stelzer, P. Torrielli, M. Zaro, JHEP 07, 079 (2014). arXiv:1405.0301 [hep-ph]

122. R.D. Ball et al. [NNPDF], Eur. Phys. J. C 77(10), 663 (2017). arXiv:1706.00428 [hep-ph]

123. A. Buckley, J. Ferrando, S. Lloyd, K. Nordström, B. Page, M. Rüfenacht, M. Schönherr, G. Watt, Eur. Phys. J. C 75, 132 (2015). arXiv: 1412.7420 [hep-ph]

124. S. Dimopoulos, H. Georgi, Nucl. Phys. B 193, 150 (1981)

125. N. Sakai, Zeit. f. Phys. C11, 153 (1981)

126. N. Polonsky, A. Pomarol, Phys. Rev. Lett. 73, 2292 (1994)

127. D.I. Kazakov, M.Y. Kalmykov, I.N. Kondrashuk, A.V. Gladyshev, Nucl. Phys. B 471, 389 (1996)

128. J.W.F. Valle, PoS corfu 98, 010 (1998). arXiv:hep-ph/9907222

129. M.A. Diaz, M. Hirsch, W. Porod, J.C. Romao, J.W.F. Valle, Phys. Rev. D 68, 013009 (2003). [Erratum: Phys. Rev. D 71, 059904 (2005)] arXiv:hep-ph/0302021

130. H.K. Dreiner, Adv. Ser. Direct. High Energy Phys. 21, 565 (2010). arXiv:hep-ph/9707435

131. G. Bhattacharyya, in Tegernsee 1997, Beyond the desert 1997, pp. 194-201. arXiv:hep-ph/9709395

132. B.C. Allanach, A. Dedes, H.K. Dreiner, Phys. Rev. D 60, 075014 (1999). arXiv:hep-ph/9906209

133. J.C. Romao, J.W.F. Valle, Nucl. Phys. B 381, 87 (1992)

134. D.H. Lyth, E.D. Stewart, Phys. Rev. D 53, 1784 (1996). arXiv:hep-ph/9510204

135. G.B. Gelmini, P. Gondolo, Phys. Rev. D 74, 023510 (2006). arXiv:hep-ph/0602230

136. CMS-PAS-FTR-18-017 (2018)

137. M. Cepeda et al., CERN Yellow Rep. Monogr. 7, 221-584 (2019). arXiv:1902.00134 [hep-ph]

138. M. Mangano, CERN Yellow Report CERN 2017-003-M arXiv:1710.06353 [hep-ph]

139. N. Craig, J. Hajer, Y.Y. Li, T. Liu, H. Zhang, JHEP 01, 018 (2017). arXiv:1605.08744 [hep-ph]

140. J. Hajer, Y.Y. Li, T. Liu, J.F.H. Shiu, JHEP 11, 124 (2015). arXiv:1504.07617 [hep-ph]

141. T. Golling et al., CERN Yellow Rep. (3), 441-634 (2017). arXiv:1606.00947 [hep-ph]

142. J. Leon, J. Perez-Mercader, M. Quiros, J. Ramirez-Mittelbrunn, Phys. Lett. B 156, 66 (1985)

143. S. Hamidi, J.H. Schwarz, Phys. Lett. B 147, 301 (1984)

144. D.R.T. Jones, S. Raby, Phys. Lett. B 143, 137 (1984)

145. K.S. Babu, T. Enkhbat, I. Gogoladze, Phys. Lett. B 555, 238 (2003). arXiv:hep-ph/0204246

146. S. Heinemeyer, M. Mondragón, G. Zoupanos, Phys. Lett. B 718, 1430 (2013). arXiv:1211.3765 [hep-ph]

147. S. Heinemeyer, M. Mondragón, G. Zoupanos, Int. J. Mod. Phys. Conf. Ser. 13, 118 (2012)
148. S. Heinemeyer, M. Mondragón, G. Zoupanos, Phys. Part. Nucl. 44, 299 (2013)

149. S. Heinemeyer, M. Mondragón, G. Patellis, N. Tracas, G. Zoupanos, Symmetry 10(3), 62 (2018). arXiv:1802.04666 [hep$\mathrm{ph}]$

150. S. Heinemeyer, M. Mondragón, G. Patellis, N. Tracas, G. Zoupanos, PoS CORFU 2017, 081 (2018)

151. S. Heinemeyer, M. Mondragón, N. Tracas, G. Zoupanos, Phys. Rep. 814, 1 (2019). arXiv:1904.00410 [hep-ph]

152. S. Heinemeyer, M. Mondragón, G. Patellis, N. Tracas, G. Zoupanos, PoS CORFU 2018, 077 (2019)

153. S. Heinemeyer, M. Mondragón, G. Zoupanos, SIGMA 6, 049 (2010). arXiv:1001.0428 [hep-ph]

154. S. Heinemeyer, M. Mondragón, G. Zoupanos, Fortsch. Phys. 61(11), 969 (2013). arXiv:1305.5073 [hep-ph]

155. S. Heinemeyer, M. Mondragón, N. Tracas, G. Zoupanos, Nucl. Phys. B 927, 319 (2018)

156. M. Mondragón, G. Zoupanos, Phys. Part. Nucl. Lett. 8, 173 (2011)

157. https://twiki.cern.ch/twiki/bin/view/AtlasPublic/ SupersymmetryPublicResults, https://twiki.cern.ch/twiki/ bin/view/CMSPublic/PhysicsResultsSUS

158. E. Ma, M. Mondragón, G. Zoupanos, JHEP 12, 026 (2004). arXiv:hep-ph/0407236

159. A. De Rújula, H. Georgi, S.L. Glashow, in Fifth Workshop on Grand Unification, K. Kang, H. Fried, P. Frampton eds. (World Scientific, Singapore, 1984), p. 88

160. G. Lazarides, C. Panagiotakopoulos, Q. Shafi, Phys. Lett. B 315, 325 (1993). arXiv:hep-ph/9306332

161. G. Lazarides, C. Panagiotakopoulos, Phys. Lett. B 336, 190 (1994). arXiv:hep-ph/9403317

162. E. Ma, Phys. Rev. D 36, 274 (1987)

163. N. Irges, G. Zoupanos, Phys. Lett. B 698, 146 (2011)

164. N. Irges, G. Orfanidis, G. Zoupanos, PoS CORFU 2011, 105 (2011)

165. S. Heinemeyer, E. Ma, M. Mondragón, G. Zoupanos, AIP Conf. Proc. 1200(1), 568 (2010). arXiv:0910.0501 [hep-ph]

166. S. Heinemeyer, E. Ma, M. Mondragón, G. Zoupanos, J. Phys. Conf. Ser. 259, 012097 (2010)

167. S. Heinemeyer, E. Ma, M. Mondragón, G. Zoupanos, Fortsch. Phys. 58, 729 (2010)

168. M. Mondragón, N.D. Tracas, G. Zoupanos, Phys. Lett. B 728, 51 (2014). arXiv:1309.0996 [hep-ph]

169. M. Mondragón, S. Heinemeyer, N. Tracas, G. Zoupanos, PoS CORFU2016 041 (2017)

170. M. Mondragón, N.D. Tracas, G. Zoupanos, Phys. Lett. B 728, 51 (2014)

171. S. Heinemeyer, M. Mondragón, N. Tracas, G. Zoupanos, JHEP 1808, $150(2018)$

172. K.J. Bae, H. Baer, E.J. Chun, Phys. Rev. D 89(3), 031701 (2014). https://doi.org/10.1103/PhysRevD.89.031701. arXiv:1309.0519 [hep-ph] 